\title{
An upper bound on the size of diamond-free families of sets
}

\author{
Dániel Grósz* \\ Abhishek Methuku ${ }^{\dagger}$ \\ Casey Tompkins ${ }^{\ddagger}$
}

\begin{abstract}
Let $\mathrm{La}(n, P)$ be the maximum size of a family of subsets of $[n]=\{1,2, \ldots, n\}$ not containing $P$ as a (weak) subposet. The diamond poset, denoted $\mathcal{Q}_{2}$, is defined on four elements $x, y, z, w$ with the relations $x<y, z$ and $y, z<w$. La $(n, P)$ has been studied for many posets; one of the major open problems is determining $\operatorname{La}\left(n, \mathcal{Q}_{2}\right)$. It is conjectured that $\operatorname{La}\left(n, \mathcal{Q}_{2}\right)=(2+o(1))\left(\begin{array}{c}n \\ \lfloor n / 2\rfloor\end{array}\right)$, and infinitely many significantly different, asymptotically tight constructions are known.

Studying the average number of sets from a family of subsets of $[n]$ on a maximal chain in the Boolean lattice $2^{[n]}$ has been a fruitful method. We use a partitioning of the maximal chains and introduce an induction method to show that $\mathrm{La}\left(n, \mathcal{Q}_{2}\right) \leq(2.20711+o(1))\left(\begin{array}{c}n \\ \lfloor n / 2\rfloor\end{array}\right)$, improving on the earlier bound of $(2.25+o(1))\left(\begin{array}{c}n \\ \lfloor n / 2\rfloor\end{array}\right)$ by Kramer, Martin and Young.
\end{abstract}

\section{Introduction}

Let $[n]=\{1,2, \ldots, n\}$. The Boolean lattice $2^{[n]}$ is defined as the family of all subsets of $[n]=\{1,2, \ldots, n\}$, and the $i$ th level of $2^{[n]}$ refers to the collection of all sets of size $i$. In 1928, Sperner proved the following well-known theorem.

Theorem 1.1 (Sperner [24]). If $\mathcal{F}$ is a family of subsets of $[n]$ such that no set contains another $(A, B \in \mathcal{F}$ implies $A \not \subset B)$, then $|\mathcal{F}| \leq\left(\begin{array}{c}n \\ \lfloor n / 2\rfloor\end{array}\right)$. Moreover, equality occurs if and only if $\mathcal{F}$ is a level of maximum size in $2^{[n]}$

Definition 1.2. Let $P$ be a finite poset, and $\mathcal{F}$ be a family of subsets of $[n]$. We say that $P$ is contained in $\mathcal{F}$ as a (weak) subposet if there is an injection $\varphi: P \rightarrow \mathcal{F}$ satisfying $x_{1}<_{p} x_{2} \Rightarrow \varphi\left(x_{1}\right) \subset \varphi\left(x_{2}\right)$ for every $x_{1}, x_{2} \in P . \mathcal{F}$ is called $P$-free if $P$ is not contained in $\mathcal{F}$ as a weak subposet. We define the corresponding extremal function as $\operatorname{La}(n, P):=\max \{|\mathcal{F}|: \mathcal{F}$ is $P$-free $\}$.

A $k$-chain, denoted by $P_{k}$, is defined to be the poset on the set $\left\{x_{1}, x_{2}, \ldots, x_{k}\right\}$ with the relations $x_{1} \leq x_{2} \leq \cdots \leq x_{k}$. Using the above notation, Sperner's theorem can be stated as $\operatorname{La}\left(n, P_{2}\right)=\left(\begin{array}{c}n \\ \lfloor n / 2\rfloor\end{array}\right)$. Let $\Sigma(n, k)$ denote the sum of the $k$ largest binomial coefficients of order $n$. An important generalization of Sperner's theorem due to Erdôs [10] states that $\mathrm{La}\left(n, P_{k+1}\right)=\Sigma(n, k)$. Moreover, equality occurs if and only if $\mathcal{F}$ is the union of $k$ of the largest levels in $2^{[n]}$.

Definition 1.3 (Posets $\mathcal{Q}_{2}, V$ and $\Lambda$ ). The diamond poset, denoted $\mathcal{Q}_{2}$ (or $\mathcal{D}_{2}$ or $\mathcal{B}_{2}$ ), is a poset on four elements $\{x, y, z, w\}$, with the relations $x<y, z$ and $y, z<w$. That is, $\mathcal{Q}_{2}$ is a subposet of a family of sets $\mathcal{A}$ if there are different sets $A, B, C, D \in \mathcal{A}$ with $A \subset B, C$ and $B, C \subset D$. (Note that $B$ and $C$ are not necessarily unrelated.) The $V$ poset is a poset on $\{x, y, z\}$ with the relations $x \leq y, z$; the $\Lambda$ poset is defined on $\{x, y, z\}$ with the relations $x, y \leq z$. That is, the $\Lambda$ is a subposet of a family of sets $\mathcal{A}$ if there are different sets $B, C, D \in \mathcal{A}$ with $B, C \subset D$.

The general study of forbidden poset problems was initiated in the paper of Katona and Tarján [1] in 1983. They determined the size of the largest family of sets containing neither a $V$ nor a $\Lambda$. They also gave an estimate on the maximum size of $V$-free families: $\left(1+\frac{1}{n}+o\left(\frac{1}{n}\right)\right)\left(\begin{array}{c}n \\ \lfloor n / 2\rfloor\end{array}\right) \leq \mathrm{La}(n, V) \leq\left(1+\frac{2}{n}\right)\left(\begin{array}{c}n \\ \lfloor n / 2\rfloor\end{array}\right)$. This result was later generalized by De Bonis and Katona [8] who obtained bounds for the $r$-fork poset, $V_{r}$ defined by the relations $x \leq y_{1}, y_{2}, \ldots, y_{r}$. Other posets for which La $(n, P)$ has been studied include complete two level posets, batons [25], crowns $O_{2 k}$ (cycle of length $2 k$ on two levels, asymptotically

\footnotetext{
*Department of Mathematics, University of Pisa. e-mail: groszdanielpub@gmail.com

${ }^{\dagger}$ Department of Mathematics, Central European University, Budapest, Hungary. e-mail: abhishekmethuku@gmail.com

¥Alfréd Rényi Institute of Mathematics, Hungarian Academy of Sciences. e-mail: ctompkins496@gmail.com
} 
solved except for $k \in\{3,5\}$ [15, 18]), butterfly [9], skew-butterfly [22], the N poset [12, harp posets $\mathcal{H}\left(l_{1}, l_{2}, \ldots, l_{k}\right)$, defined by $k$ chains of length $l_{i}$ between two fixed elements [14, and recently the complete 3 level poset $K_{r, s, t}[23$ among others. (See [13] for a nice survey by Griggs and Li.)

One of the first general results is due to Bukh [4] who determined the asymptotic value of $\operatorname{La}(n, P)$ for all posets whose Hasse diagram is a tree: If $T$ is a finite poset whose Hasse diagram is a tree of height $h(T) \geq 2$, then $\operatorname{La}(n, T)=(h(T)-1)\left(\begin{array}{c}n \\ \lfloor n / 2\rfloor\end{array}\right)\left(1+O\left(\frac{1}{n}\right)\right)$.

Using more general structures instead of chains for double counting, Burcsi and Nagy [5] obtained a weaker version of this theorem for general posets showing that $\mathrm{La}(n, P) \leq\left(\frac{|P|+h(P)}{2}-1\right)\left(\begin{array}{c}n \\ \lfloor n / 2\rfloor\end{array}\right)$. Later this was generalized by Chen and Li [6] and recently this general bound was improved by the authors of the present article [16].

The most investigated poset for which even the asymptotic value of $\operatorname{La}(n, P)$ has yet to be determined is the diamond $\mathcal{Q}_{2}$ which is the topic of our paper. The two middle levels of the Boolean lattice do not contain a diamond, so $\operatorname{La}\left(n, \mathcal{Q}_{2}\right) \geq(2-o(1))\left(\begin{array}{c}n \\ \lfloor n / 2\rfloor\end{array}\right)$. Czabarka, Dutle, Johnston and Székely [7] gave infinitely many asymptotically tight constructions by using random set families defined from posets based on Abelian groups. Such constructions suggest that the diamond problem is hard. Using a simple and elegant argument, Griggs, $\mathrm{Li}$ and $\mathrm{Lu}$ [14] showed that $\mathrm{La}\left(n, \mathcal{Q}_{2}\right)<2.296\left(\begin{array}{c}n \\ n / 2\end{array}\right)$. Some time after they had announced this bound, Axenovich, Manske and Martin [1] improved the upper bound to $2.283\left(\begin{array}{c}n \\ \lfloor n / 2\rfloor\end{array}\right)$. This bound was further improved to $2.273\left(\begin{array}{c}n \\ \lfloor n / 2\rfloor\end{array}\right)$ by Griggs, Li and Lu [14]. The best known upper bound on $\mathrm{La}\left(n, \mathcal{Q}_{2}\right)$ is $(2.25+o(1))\left(\begin{array}{c}n \\ \lfloor n / 2\rfloor\end{array}\right)$ due to Kramer, Martin and Young [17].

Definition 1.4. A maximal chain or, for the rest of this article, simply a chain of the Boolean lattice is a sequence of sets $\emptyset,\left\{x_{1}\right\},\left\{x_{1}, x_{2}\right\},\left\{x_{1}, x_{2}, x_{3}\right\}, \ldots,[n]$ with $x_{1}, x_{2}, x_{3} \ldots \in[n]$. We refer to $\left\{x_{1}, \ldots, x_{i}\right\}$ as the $i$ th set on the chain. In particular, we refer to $\left\{x_{1}\right\}$ as the first set on the chain, or just say that the chain starts with the element $x_{1}$ (as a singleton). We refer to $x_{i}$ as the $i$ th element added to form the chain.

Definition 1.5. The Lubell function of a family of sets $\mathcal{F} \subseteq 2^{[n]}$ is defined as

$$
l(n, \mathcal{F})=\sum_{F \in \mathcal{F}} \frac{1}{\left(\begin{array}{c}
n \\
|F|
\end{array}\right)} .
$$

The notation is shortened to just $l(\mathcal{F})$ when there is no ambiguity as to the dimension of the Boolean lattice.

Observation 1.6. The Lubell function of a family $\mathcal{F}$ is the average number of sets from $\mathcal{F}$ on a chain, taken over all $n$ ! chains. In particular, the Lubell function of a level is 1, and the Lubell function of an antichain $\mathcal{F}$ is the number of chains containing a set from $\mathcal{F}$ divided by $n$ !. The Lubell function is additive across a union of disjoint families of sets. Furthermore, $|\mathcal{F}| \leq l(\mathcal{F})\left(\begin{array}{c}n \\ \lfloor n / 2\rfloor\end{array}\right)$ ([19]).

The Lubell function was derived from the celebrated YMBL inequality which was independently discovered by Yamamoto, Meshalkin, Bollobás and Lubell. Using the Lubell function terminology, it states that

YMBL inequality (Yamamoto, Meshalkin, Bollobás, Lubell [26, 21, 3, 19]). If $\mathcal{F} \subseteq 2^{[n]}$ is an antichain, then $l(\mathcal{F}) \leq 1$.

For a poset $P$, let $\bar{l}(n, P)$ be the maximum of $l(n, \mathcal{F})$ over all families $\mathcal{F} \subseteq 2^{[n]}$ which are both $P$-free and contain the empty set. Let $\bar{l}(P)=\limsup _{n \rightarrow \infty} \bar{l}(n, P)$. Griggs, Li and Lu proved that

Lemma 1.7 (Griggs, Li and Lu [14]).

$$
\mathrm{La}\left(n, \mathcal{Q}_{2}\right) \leq\left(\bar{l}\left(\mathcal{Q}_{2}\right)+o(1)\right)\left(\begin{array}{c}
n \\
\lfloor n / 2\rfloor
\end{array}\right) .
$$

Kramer, Martin and Young used flag algebras to prove that

Lemma 1.8 (Kramer, Martin and Young [17]). $\bar{l}\left(\mathcal{Q}_{2}\right)=2.25$

thereby proving 
Theorem 1.9 (Kramer, Martin and Young [17]).

$$
\operatorname{La}\left(n, \mathcal{Q}_{2}\right) \leq(2.25+o(1))\left(\begin{array}{c}
n \\
\lfloor n / 2\rfloor
\end{array}\right) .
$$

The following construction shows that $\bar{l}\left(\mathcal{Q}_{2}\right) \geq 2.25$ in Lemma 1.8 There are other constructions known as well.

Example 1.10. Let $\mathcal{F} \subseteq 2^{[n]}$ consist of all the sets of the following forms: $\emptyset,\{e\},\{e, o\},\left\{o_{1}, o_{2}\right\}$ where $e$ denotes any even number in $[n]$, and $o, o_{1}$ and $o_{2}$ denote any odd numbers in $[n]$. This family is diamond-free, and $l(\mathcal{F})=2.25 \pm o(1)$.

Example 1.11. This construction is a generalization of the previous one. Let $A \subseteq[n]$ with $|A|=a n$. Let $\mathcal{F} \subseteq 2^{[n]}$ consist of all the sets of the following forms: $\emptyset,\{e\},\{e, o\},\left\{o_{1}, o_{2}\right\}$ where now $e$ denotes any element of $A$, while $o, o_{1}$ and $o_{2}$ denote any elements of $[n] \backslash A$. This family is diamond-free, and $l(\mathcal{F})=2+a-a^{2} \pm o(1)$. This family contains all size 2 sets that do not form a diamond with $\emptyset$ and the singletons, so all maximal diamond-free families on levels 0,1 and 2 that contain $\emptyset$ are of this form.

The following restriction of the problem of diamond-free families has been investigated: How big can a diamond-free family be if it can only contain sets from the middle three levels of $2^{[n]}$ (denoted $\left.\mathcal{B}(n, 3)\right)$ ? Better bounds are known with this restriction. Axenovich, Manske and Martin showed that

Theorem 1.12 (Axenovich, Manske and Martin [1]). If $\mathcal{F} \subseteq \mathcal{B}(n, 3)$ is diamond-free, then $|\mathcal{F}| \leq$ $(2.20711+o(1))\left(\begin{array}{c}n \\ \lfloor n / 2\rfloor\end{array}\right)$.

Later, Manske and Shen improved it to $2.1547\left(\begin{array}{c}n \\ \lfloor n / 2\rfloor\end{array}\right)$ in [20] and recently, Balogh, Hu, Lidický and Liu gave the best known bound of $2.15121\left(\begin{array}{c}n \\ \lfloor n / 2\rfloor\end{array}\right)$ in [2] using flag algebras.

Definition 1.13. We call a chain maximal-non-maximal (MNM) with respect to (w.r.t.) $\mathcal{F}$ if it contains a set from $\mathcal{F}$, and the biggest set contained in $\mathcal{F}$ on the chain is not maximal in $\mathcal{F}$ (i.e., there are other sets from $\mathcal{F}$ containing it on some other chains).

It is easy to see that an $\emptyset$-free family is $\Lambda$-free if and only if the family we get by adding $\emptyset$ is diamond-free; adding $\emptyset$ increases the Lubell function by 1 . In Section 2 of this paper, we prove the following lemma:

Lemma 1.14. Let $\mathcal{F} \subseteq 2^{[n]}$ be a $\Lambda$-free family that does not contain the empty set, nor any set of size bigger than $n-n^{\prime}$ for some $n^{\prime} \in \mathbf{N}$ (that can be chosen independently of $n$ ). Assume that there are $c n$ ! $M N M$ chains w.r.t. $\mathcal{F}$. Then $l(\mathcal{F}) \leq 1-\min \left(c+\frac{1}{n^{\prime}}, \frac{1}{4}\right)+\sqrt{\min \left(c+\frac{1}{n^{\prime}}, \frac{1}{4}\right)}+\frac{3}{n^{\prime}}$.

It is easy to see that in Example 1.11 the number of MNM-chains is approximately $a^{2} n$ ! (so $a \approx \sqrt{c}$ ): these are the chains whose second set is $\left\{e_{1}, e_{2}\right\}$ with $e_{1}, e_{2} \in A$. Thus, this lemma is (asymptotically) sharp, and states that for a given number of MNM chains, Example1.11 cannot be beaten (with some restriction on the sizes of the sets). Barring the requirement that the topmost $n^{\prime}$ levels be empty, Lemma 1.14 is a generalization of Lemma 1.8. The proof of Lemma1.7 in 17 actually works with the restriction of Lemma 1.14 concerning the topmost sets (that there is no set of size bigger than $n-n^{\prime}$ ) with $n^{\prime}=n / 2-n^{2 / 3}$, immediately giving a new proof of Theorem 1.9. Our proof of Lemma 1.14 includes an intricate induction step and a (non-combinatorial) lemma about functions involving a lot of elementary algebra and calculus; but it does not require flag algebras, and it does not use details of the structure of $\mathcal{F}$ above the second level (except inside the induction).

Section 3 of this paper uses Lemma 1.14 to prove our main theorem:

Theorem 1.15. $\operatorname{La}\left(n, \mathcal{Q}_{2}\right) \leq\left(\frac{\sqrt{2}+3}{2}+o(1)\right)\left(\begin{array}{c}n \\ \lfloor n / 2\rfloor\end{array}\right)<(2.20711+o(1))\left(\begin{array}{c}n \\ \lfloor n / 2\rfloor\end{array}\right)$.

The proof is inspired by the proof of Theorem 1.12 (the same bound when restricted to 3 levels) as in [1, using the idea of grouping chains by the smallest set contained in $\mathcal{F}$ on a chain (as developed in 14] and [17]). 


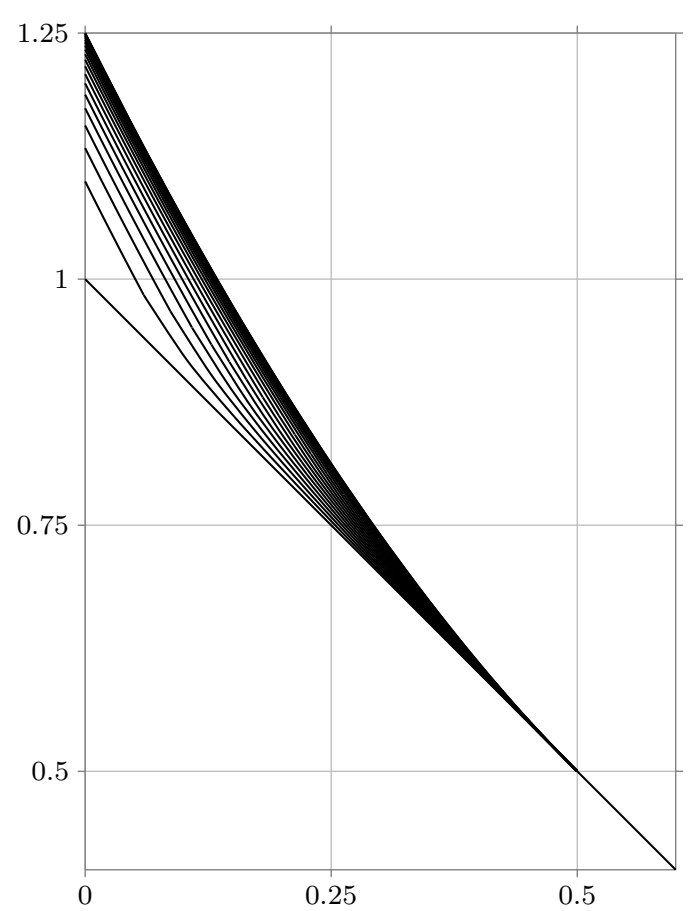

Values of $f(x, c)$ plotted in $x$, for $c=$ $0,0.0125,0.025, \ldots, 0.25$ (bottom to top). Note that the $x \geq 0.5$ part of the plots coincide.

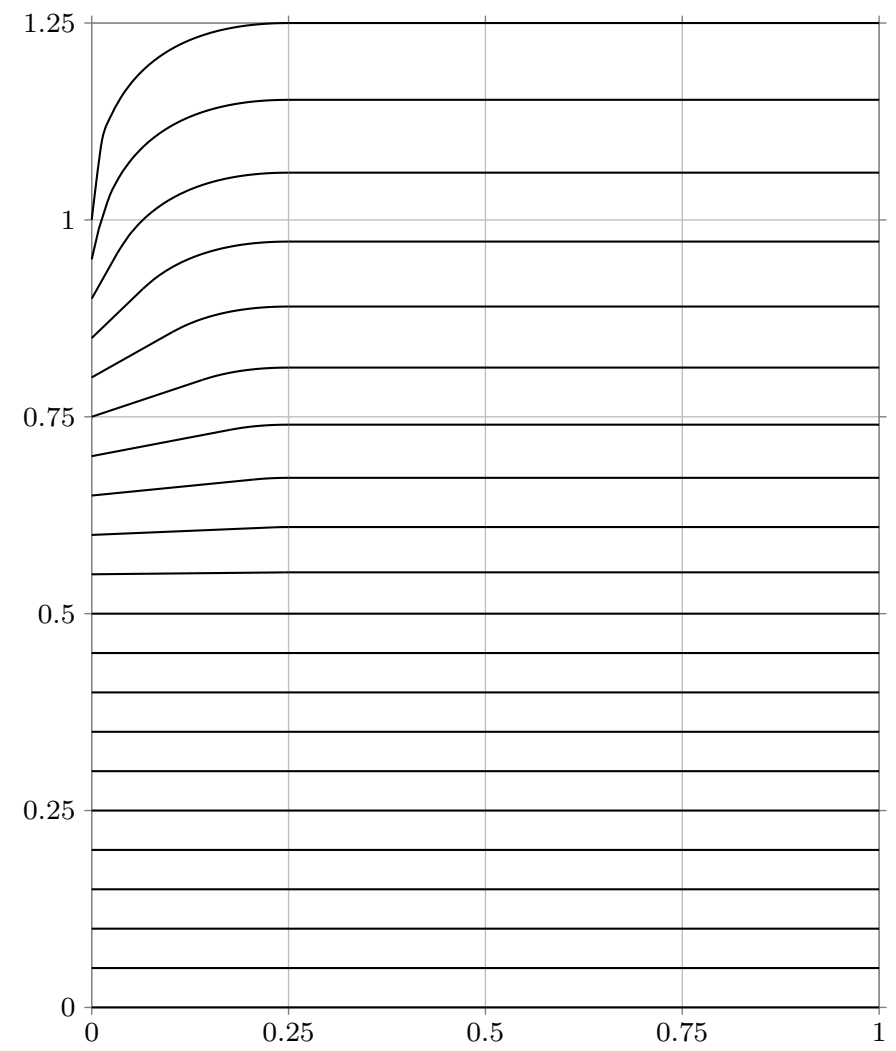

Values of $f(x, c)$ plotted in $c$, for $x=0,0.05,0.01, \ldots, 1$ (top to bottom).

\section{$2 \Lambda$-free families - Proof of Lemma 1.14}

\subsection{Definitions and main lemma}

Definition 2.1. We define the following functions:

- For $x \in[0,1], c \in[0, \infty)$,

$$
f(x, c)= \begin{cases}1-x+\left(\frac{1}{4\left(x-x^{2}\right)}-1\right) c & \text { if } x \leq \frac{1}{2} \text { and } c<4\left(x-x^{2}\right)^{2} \\ x^{2}-2 x+1-c+\sqrt{c} & \text { if } x \leq \frac{1}{2} \text { and } 4\left(x-x^{2}\right)^{2} \leq c \leq \frac{1}{4} \\ x^{2}-2 x+1.25 & \text { if } x \leq \frac{1}{2} \text { and } \frac{1}{4} \leq c \\ 1-x & \text { if } \frac{1}{2} \leq x .\end{cases}
$$

- For $x \in[0,1), c \in[0, \infty), a \in[0,1-x), \tilde{a} \in\left[0, \min \left(a, \frac{c}{x+a}\right)\right]$,

$$
g(x, c, a, \tilde{a})=a+(1-x-a) f\left(x+a, \frac{c-\tilde{a}(x+a)}{1-x-a}\right)+2 \tilde{a}(1-x-a) .
$$

- For $x \in[0,1), c \in[0, \infty), a \in[0,1-x), \tilde{a} \in\left[0, \min \left(a, \frac{c}{x+a}-x\right)\right]$,

$$
h(x, c, a, \tilde{a})=a+(1-x-a) f\left(x+a, \frac{c-(x+\tilde{a})(x+a)}{1-x-a}\right)+2 \tilde{a}(1-x-a)+x-3 x(x+a) .
$$

Lemma 2.2. The functions above satisfy the following conditions:

1. For all $c \in[0, \infty)$, if $\tilde{c}=\min \left(c, \frac{1}{4}\right)$, then $f(0, c)=1-\tilde{c}+\sqrt{\tilde{c}}$. 
2. $f(x, c)$ is concave and monotonously increasing in $c$, and monotonously decreasing in $x$.

3. For all $x \in[0,1], c \in[0, \infty), a \in[0,1-x), \tilde{a} \in\left[0, \min \left(a, \frac{c}{x+a}\right)\right]: g(x, c, a, \tilde{a}) \leq f(x, c)$.

4. For all $x \in[0,1], c \in[0, \infty), a \in[0,1-x), \tilde{a} \in\left[0, \min \left(a, \frac{c}{x+a}-x\right)\right]: h(x, c, a, \tilde{a}) \leq f(x, c)$.

5. For all $c \in[0, \infty): 1-x \leq f(x, c)$.

We prove Lemma 2.2 in Appendix A

Rather that proving Lemma 1.14 directly, we prove a strengthening of it - Lemma 2.3. This strengthened version involves additional parameters, $X$ and $\mathcal{X}$, and their functions $x, \alpha$ and $\mu$, which we introduce in order to make the inductive proof possible. Lemma 1.14 is a special case of Lemma 2.3 with $X=\mathcal{X}=\emptyset$. In the rest of Section 2, we prove Lemma 2.3.

Lemma 2.3. Let $\mathcal{F} \subseteq 2^{[n]}$ be a $\Lambda$-free family which does not contain $\emptyset$, nor any set larger than $n-n^{\prime}$ for some $n^{\prime} \in \mathbf{N}$. Let us assume that we are given a "forbidden" set $X \subseteq[n]$, with $x=\frac{|X|}{n}$. Also, let $\mathcal{X} \subset 2^{[n]}$ be a "forbidden" antichain in which each set contains exactly one element of $X$ (and may or may not be a singleton). Let us assume that the sets in $\mathcal{F}$ are disjoint from $X$, and unrelated to every set in $\mathcal{X}$. Let $\alpha=l(\mathcal{X})$, and let $\mu n$ ! be the number of chains which start with an element of $X$ as a singleton, but do not contain any set in $\mathcal{X}$. Assume, furthermore, that there are cn! MNM chains w.r.t. $\mathcal{F}$. Then $l(\mathcal{F}) \leq f\left(x, c+\mu+\frac{1}{n^{\prime}}\right)-(\alpha-\mu-x)+\frac{3}{n^{\prime}}$.

First we verify the base case of the induction.

Proposition 2.4. Lemma 2.3 holds for $n \leq n^{\prime}$.

Proof. $\mathcal{F}=\emptyset . \mathcal{X}$ is an antichain, so, by the YMBL inequality, $\alpha \leq 1$. By Lemma 2.2 Point 2 and Point 5] $f\left(x, c+\mu+\frac{1}{n^{\prime}}\right)-(\alpha-\mu-x)+\frac{3}{n^{\prime}} \geq f(x, c+\mu)-(\alpha-\mu-x) \geq 1-x-(\alpha-x) \geq 0=l(\mathcal{F})$.

From now on we assume $n^{\prime} \leq n-1$.

Notation. Let $A \subseteq[n] \backslash X$ be the set of elements of $[n]$ that appear as singletons in $\mathcal{F}$, and let $a=\frac{|A|}{n}$. Let $\mathcal{B}$ be the family of those sets in $\mathcal{F}$ which contain at least one element of $A$, but which are not singletons. Let $\beta$ be the Lubell function of $\mathcal{B}$. Let $\mathcal{C}$ be the family of those sets in $\mathcal{F}$ which only contain elements of $[n] \backslash X \backslash A$.

Let $\tilde{A}=\{e \in A:(\exists B \in \mathcal{B}: e \in B)\}$, and let $\tilde{a}=\frac{|\tilde{A}|}{n}$. Let $c_{0} n$ ! be the number of chains that start with $\{e\}$ as a singleton for some $e \in \tilde{A}$, but do not contain any set from $\mathcal{B}$. Let $\nu n$ ! be the number of chains that start with $\{e\}$ as a singleton for some $e \in \tilde{A}$, continue with an element of $[n] \backslash X \backslash A$ as the second element added to form the chain, yet do not contain any set from $\mathcal{B}$.

Let $\overline{1}=\frac{n}{n-1}>1$ and $\underline{1}=\frac{(x+a) n-1}{(x+a)(n-1)} \leq 1$. These correction factors will account for the difference from the asymptotic behavior. (They are both typically close to 1 . If $x+a=0$, let $\underline{1}=1$; it is irrelevant as it will always be multiplied by $x+a$.)

Outline of the proof: In Subsection 2.2, we make some observations on the structure of $\mathcal{X}$ and $\mathcal{B}$. In Subsection 2.4 we will finish the proof by applying induction to the Boolean lattices $\left[\left\{o_{i}\right\},[n]\right]$ where $o_{i} \in[n] \backslash X \backslash A$. When applying Lemma 2.3 by induction, we will use $X \cup A$ in the place of $X$, while sets from $\mathcal{X}$ and $\mathcal{B}$ will contribute to the family we use in the place of $\mathcal{X}$ (which we will denote by $\mathcal{X}_{i}^{\prime}$ ). We know little about the parameters of each $\mathcal{X}_{i}^{\prime}$, but we will be able to bound their sums. The relevant calculations are done in Subsection 2.3

\subsection{On the structure of $\mathcal{X}$ and $\mathcal{B}$}

Proposition 2.5. Every $D \in \mathcal{X}$ is of the form $\left\{d, o_{1}, \ldots, o_{k}\right\}$ with $d \in X, o_{1}, \ldots, o_{k} \in[n] \backslash X \backslash A$ (where $k$ may be 0).

Proof. $D$ contains exactly one element of $X$ by definition. Let $e \in A$; then $e \notin D$ for otherwise $D$ and $\{e\} \in \mathcal{F}$ would be related. 


\section{Summary of notation}

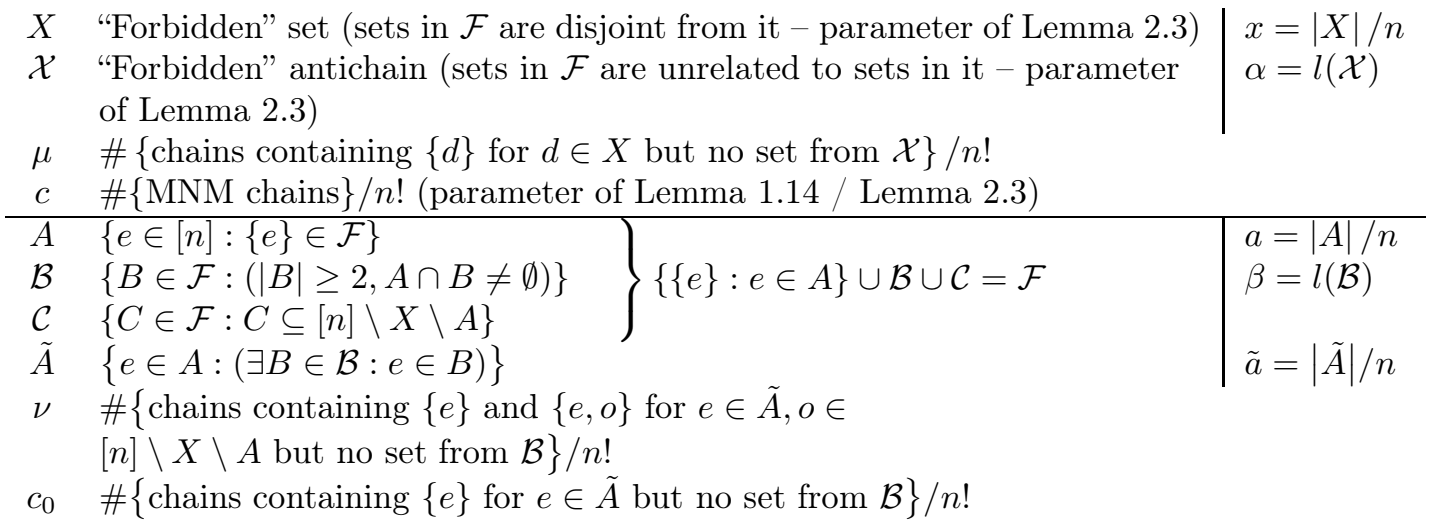

Proposition 2.6. Sets in $\mathcal{B}$ only contain one element of $A . \mathcal{B}$ is an antichain, and the sets in $\mathcal{B}$ are also unrelated to every set in $\mathcal{C}$.

Proof. If $e_{1} \in B \in \mathcal{B}$ with $e_{1} \in A$, and B was related to another set $S \in \mathcal{F}$, then $\left\{e_{1}\right\}, B$ and $S$ would form a $\Lambda$. This applies to any $S \in \mathcal{B} \cup \mathcal{C}$, as well as $S=\left\{e_{2}\right\}$ for any $e_{1} \neq e_{2} \in A$.

Proposition 2.7. $\tilde{a}(x+a) \underline{1} \leq \tilde{a}(x+a) \underline{1}+\nu=c_{0} \leq c$, and thus $\tilde{a} \leq \frac{c}{(x+a) \underline{1}}$.

Proof. Any chain on which the singleton is $\{e\}$ and the second set is $\{e, d\}$ with $e \in \tilde{A}$ and $d \in X \cup A$ is always an MNM chain: $\{e, d\}$ and any set that contains it is forbidden from being in $\mathcal{B}$ either because it is not disjoint from $X$ (when $d \in X$ ), or because it would contain two elements of $A$ (when $d \in A$ ). The number of such chains is $\tilde{a} n \cdot(a n+x n-1) \cdot(n-2) !=\tilde{a}(x+a) n ! \underline{1}$. And out of the chains which start with $\{e\}$, and whose second set is $\{e, o\}$ with some $o \in[n] \backslash X \backslash A, \nu n$ ! do not contain any set from $\mathcal{B}$.

We have $c_{0} \leq c$ because a chain whose first set is $\{e\}$ for some $e \in \tilde{A}$, but does not contain any set from $\mathcal{B}$, is an MNM chain.

For a family of sets $\mathcal{A} \subseteq 2^{[n]}$, let $m(\mathcal{A}) n$ ! be the number of chains which start with an element of $X$ as a singleton and do not contain any set from $\mathcal{A}$. (For example, $m(\mathcal{X})=\mu$, and therefore $l(\mathcal{X})-m(\mathcal{X})=$ $\alpha-\mu$.) For a fixed $d \in X$, let $m_{d}(\mathcal{A}) n$ ! be the number of chains on which the singleton is $\{d\}$, and do not contain any element of $\mathcal{A}$.

Proposition 2.8. For any $d \in X$, let $\mathcal{X}_{d}=\{D \in \mathcal{X}: d \in D\}$. We can assume without loss of generality that for any $d_{1}, d_{2} \in X,\left\{D \backslash\left\{d_{1}\right\}: D \in \mathcal{X}_{d_{1}}\right\}=\left\{D \backslash\left\{d_{2}\right\}: D \in \mathcal{X}_{d_{2}}\right\}$. That is, if $\mathcal{X}$ does not satisfy this condition, we show a family $\hat{\mathcal{X}}$ which does, and also satisfies the conditions of Lemma [2.3's statement (each set contains exactly one element of $X$, the sets are unrelated to each other and to every set in $\mathcal{F}$ ), and for which $f\left(x, c+m(\hat{\mathcal{X}})+\frac{1}{n^{\prime}}\right)-(l(\hat{\mathcal{X}})-m(\hat{\mathcal{X}})-x) \leq f\left(x, c+\mu+\frac{1}{n^{\prime}}\right)-(\alpha-\mu-x)$.

Proof. Let $d_{0} \in X$ be such that

$$
\begin{aligned}
& f\left(x, c+|X| m_{d_{0}}\left(\mathcal{X}_{d_{0}}\right)+\frac{1}{n^{\prime}}\right)-\left(|X| l\left(\mathcal{X}_{d_{0}}\right)-|X| m_{d_{0}}\left(\mathcal{X}_{d_{0}}\right)-x\right) \\
= & \min _{d \in X}\left[f\left(x, c+|X| m_{d}\left(\mathcal{X}_{d}\right)+\frac{1}{n^{\prime}}\right)-\left(|X| l\left(\mathcal{X}_{d}\right)-|X| m_{d}\left(\mathcal{X}_{d}\right)-x\right)\right] .
\end{aligned}
$$

Let $\hat{\mathcal{X}}=\left\{D \backslash\left\{d_{0}\right\} \cup\{d\}: d \in X, D \in \mathcal{X}_{d_{0}}\right\}$.

$\mathcal{X}=\bigsqcup_{d \in X} \mathcal{X}_{d}$, so $\alpha=\sum_{d \in X} l\left(\mathcal{X}_{d}\right)$. It immediately follows from the definition of $\mathcal{X}_{d}$ that if a chain has $\{d\}$ as a singleton, and does not contain any set from $\mathcal{X}_{d}$, then it does not contain any set from $\mathcal{X}$. So $\mu=\sum_{d \in X} m_{d}\left(\mathcal{X}_{d}\right)$. Similarly, $l(\hat{\mathcal{X}})=|X| l\left(\mathcal{X}_{d_{0}}\right)$ and $m(\hat{\mathcal{X}})=|X| m_{d_{0}}\left(\mathcal{X}_{d_{0}}\right)$. Since $f(x, c)$ is 
monotonously increasing and concave in $c$, using Jensen's inequality

$$
\begin{gathered}
f\left(x, c+|X| m_{d_{0}}\left(\mathcal{X}_{d_{0}}\right)+\frac{1}{n^{\prime}}\right)-\left(|X| l\left(\mathcal{X}_{d_{0}}\right)-|X| m_{d_{0}}\left(\mathcal{X}_{d_{0}}\right)-x\right) \\
\leq \frac{1}{|X|}\left[\sum_{d \in X} f\left(x, c+|X| m_{d}\left(\mathcal{X}_{d}\right)+\frac{1}{n^{\prime}}\right)-\left(|X| \sum_{d \in X} l\left(\mathcal{X}_{d}\right)-|X| \sum_{d \in X} m_{d}\left(\mathcal{X}_{d}\right)-|X| x\right)\right] \\
\leq f\left(x, c+\mu+\frac{1}{n^{\prime}}\right)-(\alpha-\mu-x) .
\end{gathered}
$$

Sets in $\hat{\mathcal{X}}$ contain exactly one element of $X$, and form an antichain. They are also unrelated to every set $S \in \mathcal{F}: S$ cannot contain any element of $X$, so it could only be related to a set in $\hat{\mathcal{X}}$ by being its subset. But $S$ must also be unrelated to every $D \in \mathcal{X}_{d_{0}} \subseteq \mathcal{X}$, so it cannot be a subset of $D \backslash\left\{d_{0}\right\} \cup\{d\}$ either.

In fact we will only use the following simple corollary of Proposition 2.8. In many parts of the rest of this section we will treat the two cases of the corollary below separately.

Corollary 2.9. With the assumption of Proposition 2.8 ,

- either $\mathcal{X}=\{\{d\}: d \in X\}$ (we refer to it as the singletons case),

- or $\mathcal{X}$ does not contain any singleton (referred to as the no singleton case).

Proof. Let $d_{1} \in X$. (If $X=\emptyset$, both statements trivially hold.) If $\left\{d_{1}\right\} \in \mathcal{X}_{d_{1}}=\left\{D \in \mathcal{X}: d_{1} \in D\right\}$, then $\mathcal{X}_{d_{1}}=\left\{\left\{d_{1}\right\}\right\}$, because sets in $\mathcal{X}_{d_{1}}$ are unrelated. So either $\mathcal{X}_{d_{1}}=\left\{\left\{d_{1}\right\}\right\}$ or $\mathcal{X}_{d_{1}}$ does not contain any singleton, yielding the two cases above by Proposition 2.8

Remark. The fact that sets in $\mathcal{X}$ contain an element of $X$ implies that sets in $\mathcal{F}$ do not contain sets in $\mathcal{X}$. Now, let us consider what restrictions are imposed on $\mathcal{F}$ by the fact that sets in $\mathcal{F}$ are not contained in the sets in $\mathcal{X}$, beyond the other conditions of Lemma 2.3 (namely that all the sets in $\mathcal{F}$ are disjoint from $X)$.

In the singletons case, clearly there are no such additional restrictions. However, in the no singleton case, there are two additional restrictions that are not already implied by the set $X$ :

- The union of singletons in $\mathcal{F}, A \subseteq[n] \backslash \bigcup \mathcal{X}$.

- Sets in $\mathcal{C}$ must not be contained in sets in $\mathcal{X}$. Clearly this imposes a restriction only if $\mathcal{X}$ contains sets bigger than 2 .

Example 2.10. Let $C \subseteq[n] \backslash X$, and let $\mathcal{X}=\{\{d, o\}: d \in X, o \in C\}$. Then $\alpha=l(\mathcal{X})=\frac{2 \cdot x n \cdot|C| \cdot(n-2) !}{n !}=$ $2 x \frac{|C|}{n} \overline{1}$, and $\mu=\frac{x n \cdot(x n+a n-1) \cdot(n-2) !}{n !}=x(x+a) \underline{1}$. The only restriction on $\mathcal{F}$ that this $\mathcal{X}$ creates is that the union of singletons $A \subseteq[n] \backslash X \backslash C$.

In other words, let us assume that $\alpha=l(\mathcal{X})=2 x \gamma \overline{1}$ for $\gamma \in \mathbf{R}$ (without assuming that $\mathcal{X}$ is of the above form). Then it is possible that $a=\frac{|A|}{n}$ can be as big as $1-x-\gamma$ with $\mathcal{X}$ not creating any restrictions on $\mathcal{C}$ (depending on the actual structure of $\mathcal{X}$, namely, if it is made up of sets of size 2 as above; then $\alpha=2 x(1-x-a) \overline{1}$ and $\mu=x(x+a) \underline{1})$. But if $a>1-x-\gamma$, then $\alpha=2 x \gamma \overline{1}$ implies that $\mathcal{X}$ contains sets bigger than 2 , and thus it creates restrictions on $\mathcal{C}$. So, in the no singleton case, one way to understand the calculations that follow is to check them for $\mathcal{X}=\{\{d, o\}: d \in X, o \in C\}$; then check what happens if $x, c$ and $a$ are fixed, but $\mathcal{X}$ is changed.

\subsection{Chain calculations}

Now we estimate the numbers of certain types of chains, in preparation for applying induction.

Proposition 2.11. In the no singleton case, $(\alpha-x+\mu) n$ ! chains start with $\{o\}$ for some $o \in[n] \backslash X \backslash A$, and contain a set from $\mathcal{X}$.

Proof. A total of $\alpha n$ ! chains contain a set $D \in \mathcal{X}$. By Proposition 2.5, the singleton on such a chain is either from $X$ or $[n] \backslash X \backslash A$. The number of chains which start with an element of $X$ as their singleton and do not contain a set from $\mathcal{X}$ is $\mu n$ !, so the number of chains which contain a set from $\mathcal{X}$, and which start with an element of $X$, is $(x-\mu) n$ !. On the rest, the singleton is from $[n] \backslash X \backslash A$. 
Proposition 2.12. $(\beta-\tilde{a}(1-x-a) \overline{1}+\nu) n$ ! chains start with $\{o\}$ for some $o \in[n] \backslash X \backslash A$, and contain a set from $\mathcal{B}$.

Proof. A total of $\beta n$ ! chains contain a set from $\mathcal{B}$. A set in $\mathcal{B}$ is of the form $\left\{e, o_{1}, \ldots, o_{k}\right\}$ with $e \in$ $\tilde{A}, o_{1}, \ldots, o_{k} \in[n] \backslash X \backslash A, k \geq 1$. A chain that contains a $B \in \mathcal{B}$, and does not start with $\{o\}$ for some $o \in[n] \backslash X \backslash A$, must start with an element of $\tilde{A}$, and continue with an element of $[n] \backslash X \backslash A$ as the second element added to form the chain. There are $\tilde{a} n \cdot(1-x-a) n \cdot(n-2) !=\tilde{a}(1-x-a) \overline{1} n$ ! such chains, out of which $\nu n$ ! do not contain any set from $\mathcal{B}$. So $(\tilde{a}(1-x-a) \overline{1}-\nu) n$ ! chains contain a set from $\mathcal{B}$ and start with an element of $\tilde{A}$. The rest start with $\{o\}$ for some $o \in[n] \backslash X \backslash A$.

Proposition 2.13. In the no singleton case, $\mu \geq x(x+a) \underline{1}$; and the number of chains of the form $\emptyset,\{d\},\{d, o\}, \ldots$ with $d \in X, o \in[n] \backslash X \backslash A$, which do not contain any set from $\mathcal{X}$, is $(\mu-x(x+a) \underline{1}) n !$.

Proof. A total of $\mu n$ ! chains start with an element of $X$ and do not contain any set from $\mathcal{X}$. The chains of the form $\emptyset,\left\{d_{1}\right\},\left\{d_{1}, d_{2}\right\}, \ldots$ with $d_{1} \in X, d_{2} \in X \cup A$ never contain a set from $\mathcal{X}$ when $\mathcal{X}$ contains no singleton. The number of these chains is $x n \cdot(x n+a n-1) \cdot(n-2) !=(x(x+a) \underline{1}) n !$. For the rest, the second element added to form the chain is from $[n] \backslash X \backslash A$.

Notation. Let $X^{\prime}=X \cup A$. Let $\mathcal{Y}=\{\{d, o\}: d \in X, o \in[n] \backslash X \backslash A\}$, and let $\mathcal{Z}=\{\{e, o\}: e \in A \backslash \tilde{A}, o \in$ $[n] \backslash X \backslash A\}\}$. In the singletons case, let $\mathcal{X}^{\prime}=\mathcal{Y} \sqcup \mathcal{B} \sqcup \mathcal{Z}$. (Note that here and in the rest of the paper, $\sqcup$ stands for a union of sets which are pairwise disjoint.) In the no singleton case, let $\mathcal{X}^{\prime}=\mathcal{X} \sqcup \mathcal{B} \sqcup \mathcal{Z}$.

Proposition 2.14. The three families which make up $\mathcal{X}^{\prime}$ are indeed disjoint in each case, and their union forms an antichain.

Proof. $\mathcal{B}$ is an antichain by Proposition 2.6, $\mathcal{X}$ is an antichain by definition; and $\mathcal{Y}$ and $\mathcal{Z}$ are antichains because both consist of size 2 sets only. Let $D=\left\{d_{\tilde{A}}, o_{1}, \ldots, o_{k}\right\}_{\tilde{A}} \in \mathcal{X}, Y=\{d, o\} \in \mathcal{Y}$, $B=\left\{e_{1}, p_{1}, \ldots, p_{l}\right\} \in \mathcal{B}$ and $Z=\left\{e_{2}, q\right\} \in \mathcal{Z}$ with $d \in X, e_{1} \in \tilde{A}, e_{2} \in A \backslash \tilde{A}, o_{i}, o, p_{i}, q \in[n] \backslash X \backslash A$, and $l \geq 1 . B$ is unrelated to $D$ by definition, and to $Y$ because $d \notin B$ and $|B| \geq 2$. $Z$ is unrelated to $D$ and $Y$ because $d \notin Z$ and $e_{2} \notin D, Y ; Z$ is unrelated to $B$ because $e_{1} \notin Z$ and $e_{2} \notin B$.

Proposition 2.15. Sets in $\mathcal{C}$ are disjoint from $X^{\prime}$, and they are unrelated to every set in $\mathcal{X}^{\prime}$ (in both cases).

Proof. For every $C \in \mathcal{C}, C \subseteq[n] \backslash X^{\prime}$ and it is unrelated to every set in $\mathcal{X}$ by definition. $C$ is unrelated to every set in $\mathcal{B}$ by Proposition 2.6. It also cannot be a superset of a $Y \in \mathcal{Y}$ or a $Z \in \mathcal{Z}$, since those contain an element of $X$ or $A$; neither a proper subset of $Y$ or $Z$ because $|Y|=|Z|=2 \leq|C|$.

Proposition 2.16. The number of chains that start with an element of $[n] \backslash X^{\prime}$ and contain a set from $\mathcal{X}^{\prime}$ is

- at least $[x(1-x-a) \overline{1}+(\beta-\tilde{a}(1-x-a) \overline{1}+\nu)+(1-x-a)(a-\tilde{a}) \overline{1}] n$ ! in the singletons case, and

- at least $[(\alpha-x+\mu)+(\beta-\tilde{a}(1-x-a) \overline{1}+\nu)+(1-x-a)(a-\tilde{a}) \overline{1}] n$ ! in the no singleton case.

Proof. The number of chains on which the singleton is $\{o\}$ with $o \in[n] \backslash X^{\prime}=[n] \backslash X \backslash A$, and the second set is $\{o, d\} \in \mathcal{Y}$ with $d \in X$, is $x n \cdot(1-x-a) n \cdot(n-2) !=x(1-x-a) \overline{1} n !$. The number of chains on which the singleton is $\{o\}$, and the second set is $\{o, e\} \in \mathcal{Z}$ with $e \in A \backslash \tilde{A}$, is $(1-x-a) n \cdot(a-\tilde{a}) n \cdot(n-2) !=$ $(1-x-a)(a-\tilde{a}) \overline{1} n$ !. The rest follows from Proposition 2.11 and Proposition 2.12 .

Proposition 2.17. The number of chains on which the singleton is $\{o\}$ with $o \in[n] \backslash X^{\prime}$, the second set is $\{o, d\}$ with $d \in X^{\prime}=X \cup A$, and which do not contain any set from $\mathcal{X}^{\prime}$, is

- $\nu n$ ! in the singletons case, and

- $(\mu-x(x+a) \underline{1}+\nu) n$ ! in the no singleton case. 
Proof. Let $\mathcal{A}=\emptyset, A_{1}, A_{2}, \ldots, A_{n-1},[n]$ be a chain with $\emptyset \subset A_{1} \subset A_{2} \subset \ldots \subset A_{n-1} \subset[n]$. Let $\varphi(\mathcal{A})$ be the chain $\emptyset, A_{2} \backslash A_{1}, A_{2}, A_{3}, \ldots, A_{n-1},[n]$. (In other words, in the order in which elements of $[n]$ are added to form the chain, the first two are swapped.) $\varphi$ is a bijection.

It is easy to check that $\mathcal{X}^{\prime}$ does not contain singletons. $\varphi$ is a bijection between chains of the form $\emptyset,\{o\},\{o, d\}, \ldots$ containing no set from $\mathcal{X}^{\prime}$, and chains of the form $\emptyset,\{d\},\{o, d\}, \ldots$ containing no set from $\mathcal{X}^{\prime}$, with $o \in[n] \backslash X^{\prime}$ and $d \in X \cup A$. Below we classify the chains $\emptyset,\{d\},\{o, d\}, \ldots$ based on what set $d$ belongs to and count them separately.

- For $d \in X,\{o, d\} \in \mathcal{Y}$ in the singletons case. In the no singleton case, $(\mu-x(x+a) \underline{1}) n$ ! chains of the form $\emptyset,\{d\},\{o, d\}, \ldots$ contain no set from $\mathcal{X}$ by Proposition 2.13, these chains also contain no set from $\mathcal{B}$ or $\mathcal{Z}$, since sets from those do not contain any element of $X$.

- For $d \in \tilde{A}$, the number of chains of this form which contain no set from $\mathcal{B}$ is $\nu n$ !; these chains also contain no set from $\mathcal{X}, \mathcal{Y}$ or $\mathcal{Z}$, since sets from those contain no element of $\tilde{A}$.

- For $d \in A \backslash \tilde{A},\{o, d\} \in \mathcal{Z}$.

Summing these cases, we get the statement of the proposition.

\subsection{Inductive step}

Notation. Using standard notation for intervals, let $[A,[n]]$ denote the Boolean lattice $\{S \subseteq[n]: A \subseteq S\}$. Let $[n] \backslash X^{\prime}=[n] \backslash X \backslash A=\left\{o_{1}, o_{2}, \ldots, o_{(1-x-a) n}\right\}$; and for a family of sets $\mathcal{A}$, let $\mathcal{A}-o_{i}=\left\{S \backslash\left\{o_{i}\right\}: S \in \mathcal{A}\right\}$. Let $\mathcal{C}_{i}^{\prime}=\left(\mathcal{C} \cap\left[\left\{o_{i}\right\},[n]\right]\right)-o_{i}$, and $\mathcal{X}_{i}^{\prime}=\left(\mathcal{X}^{\prime} \cap\left[\left\{o_{i}\right\},[n]\right]\right)-o_{i}$. Let $\alpha_{i}^{\prime}=l\left(n-1, \mathcal{X}_{i}^{\prime}\right)$. (Here the Lubell function on the Boolean lattice $2^{[n] \backslash\left\{o_{i}\right\}}$ of order $n-1$ is used.)

$\mathcal{C}_{i}^{\prime} \subseteq 2^{[n] \backslash\left\{o_{i}\right\}}$ is a $\Lambda$-free family which does not contain $\emptyset$ (since $o_{i} \notin A$, so $\left\{o_{i}\right\} \notin \mathcal{F}$ ), nor any set larger than $n-1-n^{\prime}$. Sets in $\mathcal{C}_{i}^{\prime}$ are disjoint from $X^{\prime}$, and are unrelated to sets in $\mathcal{X}_{i}^{\prime}$ by Proposition 2.15, Moreover, every set in $\mathcal{X}_{i}^{\prime}$ contains exactly one element of $X^{\prime}$. Therefore, the conditions of Lemma [2.3 are satisfied for the family $\mathcal{C}_{i}^{\prime} \subseteq 2^{[n] \backslash\left\{o_{i}\right\}}$ where the corresponding "forbidden" set is $X^{\prime} \subseteq[n] \backslash\left\{o_{i}\right\}$, with $\frac{\left|X^{\prime}\right|}{n-1}=(x+a) \overline{1}$ and the corresponding "forbidden" antichain is $\mathcal{X}_{i}^{\prime}$.

Since $\mathcal{X}_{i}^{\prime}$ is an antichain, $\alpha_{i}^{\prime}(n-1)$ ! is the number of chains in $2^{[n] \backslash\left\{o_{i}\right\}}$ that contain a set from $\mathcal{X}_{i}^{\prime}$. Chains of $2^{[n] \backslash\left\{o_{i}\right\}}$ correspond to chains of $2^{[n]}$ that start with $\left\{o_{i}\right\}$. So by Proposition 2.16, in the singletons case

$$
\sum_{i=1}^{(1-x-a) n} \alpha_{i}^{\prime} \geq[x(1-x-a) \overline{1}+(\beta-\tilde{a}(1-x-a) \overline{1}+\nu)+(1-x-a)(a-\tilde{a}) \overline{1}] n,
$$

and in the no singleton case

$$
\sum_{i=1}^{(1-x-a) n} \alpha_{i}^{\prime} \geq[(\alpha-x+\mu)+(\beta-\tilde{a}(1-x-a) \overline{1}+\nu)+(1-x-a)(a-\tilde{a}) \overline{1}] n .
$$

Let $\mu_{i}^{\prime}(n-1)$ ! be the number of chains in the Boolean lattice $2^{[n] \backslash\left\{o_{i}\right\}}$ which start with an element of $X^{\prime}$ as a singleton, but do not contain any set from $\mathcal{X}_{i}^{\prime}$. By Proposition 2.17 in the singletons case

$$
\sum_{i=1}^{(1-x-a) n} \mu_{i}^{\prime}=\nu n
$$

and in the no singleton case

$$
\sum_{i=1}^{(1-x-a) n} \mu_{i}^{\prime}=(\mu-x(x+a) \underline{1}+\nu) n .
$$

Let $c_{i}^{\prime}(n-1)$ ! be the number of MNM chains w.r.t. $\mathcal{C}_{i}^{\prime}$ in $2^{[n] \backslash\left\{o_{i}\right\}}$. The corresponding $2^{[n]}$-chains, starting with $\left\{o_{i}\right\}$, are MNM chains w.r.t. $\mathcal{F}$. The total number of MNM chains w.r.t. $\mathcal{F}$ is $c n$ !, out of which $c_{0} n$ ! start with an element of $A$ as a singleton. By Proposition 2.7.

$$
\sum_{i=1}^{(1-x-a) n} c_{i}^{\prime}=\left(c-c_{0}\right) n=(c-\tilde{a}(x+a) \underline{1}-\nu) n .
$$


The following two examples are typical cases where, in the induction step for the $\mathcal{C}_{i}^{\prime}$ 's, we will get the singletons case and the no singleton case respectively.

Example 2.18. Let $X=\mathcal{X}=\emptyset$ and $\mathcal{B}=\{\{e, o\}: e \in A, o \in[n] \backslash A\}$. Then $\tilde{A}=A, \beta=2 a(1-a) \overline{1}$, and $\nu=0 . X^{\prime}=A$, and $\mathcal{X}_{i}^{\prime}=\{\{e\}: e \in A\} \cdot \sum_{i=1}^{(1-a) n} \alpha_{i}^{\prime}=a(1-a) \overline{1} n, \alpha_{i}^{\prime}=a \overline{1}=\frac{\left|X^{\prime}\right|}{n-1}$ and $\mu_{i}^{\prime}=0$. $\sum_{i=1}^{(1-x-a) n} c_{i}^{\prime}=\left(c-a^{2}\right) n$ and the average of the $c_{i}^{\prime}$ 's is $\frac{c-a^{2}}{1-a}$.

Example 2.19. Let $X=\mathcal{X}=\emptyset$ and $\mathcal{B} \subseteq \hat{\mathcal{B}}:=\left\{\left\{e, o_{1}, o_{2}\right\}: e \in A, o_{1}, o_{2} \in[n] \backslash A\right\}$. Then $X^{\prime}=A$, and $\mathcal{X}_{i}^{\prime} \subseteq\left\{\{e, o\}: e \in A, o \in[n] \backslash A \backslash\left\{o_{i}\right\}\right\}$. Chains on $2^{[n]}$ of the form $\emptyset,\left\{e_{1}\right\},\left\{e_{1}, o\right\},\left\{e_{1}, o, e_{2}\right\}, \ldots$ do not intersect $\mathcal{B}$. So $\sum_{i=1}^{(1-a) n} \mu_{i}^{\prime}=\nu \geq a^{2}(1-a) \overline{1}^{2} \frac{\overline{1} a(n-1)-1}{\overline{1} a(n-2)} n$ (greater if $\mathcal{B} \varsubsetneqq \hat{\mathcal{B}}$ ), and the average of the $\mu_{i}^{\prime}$ 's is $\geq a^{2} \overline{1}^{2} \frac{\overline{1} a(n-1)-1}{\overline{1} a(n-2)}=x^{\prime 2} \frac{x^{\prime}(n-1)-1}{x^{\prime}((n-1)-1)}$ where $x^{\prime}=\frac{\left|X^{\prime}\right|}{n-1}$. In the case of $\mathcal{B}=\hat{\mathcal{B}}$, the size of the sets in $\mathcal{C}$ is at least 3 , and the size of those in $\mathcal{C}_{i}^{\prime}$ is at least 2 .

\section{Proposition 2.20.}

$$
l(\mathcal{C})=\frac{1}{n} \sum_{i=1}^{(1-x-a) n} l\left(n-1, \mathcal{C}_{i}^{\prime}\right) \quad \text { and } \quad l(\mathcal{F})=a+\beta+l(\mathcal{C})=a+\beta+\frac{1}{n} \sum_{i=1}^{(1-x-a) n} l\left(n-1, \mathcal{C}_{i}^{\prime}\right) .
$$

(Still understanding the one parameter version $l(\mathcal{F})$ as $l(n, \mathcal{F})$ for a family $\mathcal{F} \subseteq 2^{[n]}$.)

Proof. Every chain in the Boolean lattice $2^{[n]}$ that intersects $\mathcal{C}$ has an $\left\{o_{i}\right\}$ as a singleton, and thus corresponds to a chain in the Boolean lattice $\left[\left\{o_{i}\right\},[n]\right]-o_{i}$ that intersects $\mathcal{C}_{i}^{\prime}$.

$l(\mathcal{C})=\frac{1}{n !} \sum_{\mathcal{H} \text { is a chain in } 2^{[n]}}|\mathcal{H} \cap \mathcal{C}|=\frac{1}{n !} \sum_{i=1}^{(1-x-a) n} \sum_{\mathcal{H} \text { is a chain in }\left[\left\{o_{i}\right\},[n]\right]-o_{i}}\left|\mathcal{H} \cap \mathcal{C}_{i}^{\prime}\right|=\frac{1}{n} \sum_{i=1}^{(1-x-a) n} l\left(n-1, \mathcal{C}_{i}^{\prime}\right)$.

Let $\mathcal{A}=\{\{e\}: e \in A\}$. Then $\mathcal{F}=\mathcal{A} \sqcup \mathcal{B} \sqcup \mathcal{C}$. So $l(\mathcal{F})=l(\mathcal{A})+l(\mathcal{B})+l(\mathcal{C})$ with $l(\mathcal{A})=\frac{|A|}{n}=a$ and $l(\mathcal{B})=\beta$.

We now prove Lemma 2.3 (and thus Lemma 1.14) using induction on $n$. According to Proposition 2.4. Lemma 2.3 holds for $n \leq n^{\prime}$. By induction and Lemma 2.2 Point 2,

$$
\begin{aligned}
l\left(n-1, \mathcal{C}_{i}^{\prime}\right) & \leq f\left((x+a) \overline{1}, c_{i}^{\prime}+\mu_{i}^{\prime}+\frac{1}{n^{\prime}}\right)-\left(\alpha_{i}^{\prime}-\mu_{i}^{\prime}-(x+a) \overline{1}\right)+\frac{3}{n^{\prime}} \\
& \leq f\left(x+a, c_{i}^{\prime}+\mu_{i}^{\prime}+\frac{1}{n^{\prime}}\right)-\left(\alpha_{i}^{\prime}-\mu_{i}^{\prime}-(x+a) \overline{1}\right)+\frac{3}{n^{\prime}} .
\end{aligned}
$$

So, by Proposition 2.20, we have

$$
\begin{aligned}
l(\mathcal{C}) & =\frac{1}{n} \sum_{i=1}^{(1-x-a) n} l\left(n-1, \mathcal{C}_{i}^{\prime}\right) \leq \frac{1}{n} \sum_{i=1}^{(1-x-a) n} f\left(x+a, c_{i}^{\prime}+\mu_{i}^{\prime}+\frac{1}{n^{\prime}}\right) \\
& -\frac{1}{n}\left(\sum_{i=1}^{(1-x-a) n} \alpha_{i}^{\prime}-\sum_{i=1}^{(1-x-a) n} \mu_{i}^{\prime}-\sum_{i=1}^{(1-x-a) n}(x+a) \overline{1}\right)+\frac{1}{n} \cdot \frac{3(1-x-a) n}{n^{\prime}} .
\end{aligned}
$$

We handle the case of $1-x-a=0$ separately. If $1-x-a=0, A=[n] \backslash X$ and, since any nonsingleton $\left\{e_{1}, e_{2}, \ldots\right\} \in \mathcal{F}$ would form a $\Lambda$ with the singletons $\left\{e_{1}\right\},\left\{e_{2}\right\} \in \mathcal{F}$, we have $\mathcal{F}=\mathcal{A}$ and $l(\mathcal{F})=a=1-x$. This is only possible in the singletons case, since a non-singleton in $\mathcal{X}$ would have to contain elements of $[n] \backslash X \backslash A$. In the singletons case $\alpha=x$ and $\mu=0$, so $l(\mathcal{F})=1-x \leq f(x, c) \leq$ $f\left(x, c+\mu+\frac{1}{n^{\prime}}\right)-(\alpha-\mu-x)+\frac{3}{n^{\prime}}$ by Lemma 2.2 Point 5. From now on, we assume that $1-x-a>0$. Since $f$ is concave in $c$, by Jensen's inequality, and since $f$ is monotonously decreasing in $x$,

$$
\begin{aligned}
l(\mathcal{C}) & \leq(1-x-a) f\left(x+a, \frac{\sum_{i=1}^{(1-x-a) n} c_{i}^{\prime}+\sum_{i=1}^{(1-x-a) n} \mu_{i}^{\prime}}{(1-x-a) n}+\frac{1}{n^{\prime}}\right) \\
& -\left(\frac{1}{n} \sum_{i=1}^{(1-x-a) n} \alpha_{i}^{\prime}-\frac{1}{n} \sum_{i=1}^{(1-x-a) n} \mu_{i}^{\prime}-(1-x-a)(x+a) \overline{1}\right)+\frac{3(1-x-a)}{n^{\prime}} .
\end{aligned}
$$


Correction term calculations that we will use later (assuming $n^{\prime} \leq n-1$ ):

$$
\begin{gathered}
(1-\underline{1})(x+\tilde{a})(x+a)+\frac{1-x-a}{n^{\prime}}=\frac{(x+\tilde{a})(1-x-a)}{n-1}+\frac{1-x-a}{n^{\prime}} \\
\leq \frac{(1+x+\tilde{a})(1-x-a)}{n^{\prime}} \leq \frac{1-(x+a)^{2}}{n^{\prime}} \leq \frac{1}{n^{\prime}} . \\
(1-\underline{1}) \tilde{a}(x+a)+\frac{1-x-a}{n^{\prime}} \leq(1-\underline{1})(x+\tilde{a})(x+a)+\frac{1-x-a}{n^{\prime}} \leq \frac{1}{n^{\prime}} . \\
2 \tilde{a}(1-x-a)(\overline{1}-1)+2(\overline{1}-1) x-(2(\overline{1}-1)+(\underline{1}-1)) x(x+a)+\frac{3(1-x-a)}{n^{\prime}} \\
\quad \leq \frac{2 a+3 x}{n-1}+\frac{3(1-x-a)}{n^{\prime}} \leq \frac{3}{n^{\prime}} . \\
2 \tilde{a}(1-x-a)(\overline{1}-1)+\frac{3(1-x-a)}{n^{\prime}} \leq \frac{2 a}{n-1}+\frac{3(1-x-a)}{n^{\prime}} \leq \frac{3}{n^{\prime}} .
\end{gathered}
$$

In the singletons case:

$$
\begin{aligned}
l(\mathcal{F}) & \leq a+\beta+(1-x-a) f\left(x+a, \frac{(c-\tilde{a}(x+a) \underline{1}-\nu)+\nu}{1-x-a}+\frac{1}{n^{\prime}}\right) \\
& -([x(1-x-a) \overline{1}+(\beta-\tilde{a}(1-x-a) \overline{1}+\nu)+(1-x-a)(a-\tilde{a}) \overline{1}] \\
& -\nu-(1-x-a)(x+a) \overline{1})+\frac{3(1-x-a)}{n^{\prime}} \\
& =a+(1-x-a) f\left(x+a, \frac{c-\tilde{a}(x+a) \underline{1}}{1-x-a}+\frac{1}{n^{\prime}}\right)+2 \tilde{a}(1-x-a) \overline{1}+\frac{3(1-x-a)}{n^{\prime}} \\
& =a+(1-x-a) f\left(x+a, \frac{c-\tilde{a}(x+a)+(1-\underline{1}) \tilde{a}(x+a)+\frac{1-x-a}{n^{\prime}}}{1-x-a}\right)+2 \tilde{a}(1-x-a) \\
& +2 \tilde{a}(1-x-a)(\overline{1}-1)+\frac{3(1-x-a)}{n^{\prime}} .
\end{aligned}
$$

By Lemma 2.2 Point 2 and Point 3, and (2) and (4) in the Correction term calculations (note that in this case $\alpha=x$ and $\mu=0$ ),

$$
l(\mathcal{F}) \leq g\left(x, c+\frac{1}{n^{\prime}}, a, \tilde{a}\right)+\frac{3}{n^{\prime}} \leq f\left(x, c+\frac{1}{n^{\prime}}\right)+\frac{3}{n^{\prime}}=f\left(x, c+\mu+\frac{1}{n^{\prime}}\right)-(\alpha-\mu-x)+\frac{3}{n^{\prime}} .
$$

(Note that $\tilde{a} \leq \frac{c}{(x+a) \underline{1}}$, so $0 \leq \frac{c-\tilde{a}(x+a) \underline{1}}{1-x-a} \leq \frac{c+\frac{1}{n^{\prime}}-\tilde{a}(x+a)}{1-x-a}$, and $\tilde{a} \leq \frac{c+\frac{1}{n^{\prime}}}{x+a}$.)

In the no singleton case:

$$
\begin{aligned}
l(\mathcal{F}) & \leq a+\beta+(1-x-a) f\left(x+a, \frac{(c-\tilde{a}(x+a) \underline{1}-\nu)+\mu-x(x+a) \underline{1}+\nu}{1-x-a}+\frac{1}{n^{\prime}}\right) \\
& -([(\alpha-x+\mu)+(\beta-\tilde{a}(1-x-a) \overline{1}+\nu)+(1-x-a)(a-\tilde{a}) \overline{1}] \\
& -[\mu-x(x+a) \underline{1}+\nu]-(1-x-a)(x+a) \overline{1})+\frac{3(1-x-a)}{n^{\prime}} \\
& =a+(1-x-a) f\left(x+a, \frac{\left.c+\mu-(x+\tilde{a})(x+a) \underline{1}+\frac{1}{n^{\prime}}\right)-(\alpha-x(x+a) \underline{1}-x)}{1-x-a}\right) \\
& +2 \tilde{a}(1-x-a) \overline{1}+(2 \cdot \overline{1}-1) x-(2 \cdot \overline{1}+\underline{1}) x(x+a)+\frac{3(1-x-a)}{n^{\prime}} \\
& =a+(1-x-a) f\left(x+a, \frac{c+\mu-(x+\tilde{a})(x+a)+(1-\underline{1})(x+\tilde{a})(x+a)+\frac{1-x-a}{n^{\prime}}}{1-x-a}\right) \\
& +2 \tilde{a}(1-x-a)+x-3 x(x+a)-(\alpha-x(x+a) \underline{1}-x) \\
& +2 \tilde{a}(1-x-a)(\overline{1}-1)+2(\overline{1}-1) x-(2(\overline{1}-1)+(\underline{1}-1)) x(x+a)+\frac{3(1-x-a)}{n^{\prime}} .
\end{aligned}
$$


By Lemma 2.2 Point 2 and Point 4. Proposition 2.13, and (1) and (3) in the Correction term calculations,

$$
l(\mathcal{F}) \leq h\left(x, c+\mu+\frac{1}{n^{\prime}}, a, \tilde{a}\right)-(\alpha-\mu-x)+\frac{3}{n^{\prime}} \leq f\left(x, c+\mu+\frac{1}{n^{\prime}}\right)-(\alpha-\mu-x)+\frac{3}{n^{\prime}} .
$$

(Note that $\tilde{a} \leq \frac{c}{(x+a) \underline{1}}$, so $0 \leq \frac{c-\tilde{a}(x+a) \underline{1}}{1-x-a} \leq \frac{c+\mu+\frac{1}{n^{\prime}}-(x+\tilde{a})(x+a)}{1-x-a}$, and $\tilde{a} \leq \frac{c+\mu+\frac{1}{n^{\prime}}}{x+a}-x$.)

\section{Diamond-free families - Proof of Theorem 1.15}

Let $\mathcal{F}$ be a diamond-free family on $2^{[n]}$.

We cite Lemma 1 from [1]:

Lemma 3.1 (Axenovich, Manske, Martin [1]).

$$
\sum_{\substack{k \in\{0,1, \ldots, n\} \\
|k-n / 2| \geq n^{2 / 3}}}\left(\begin{array}{l}
n \\
k
\end{array}\right) \leq 2^{n-\Omega\left(n^{1 / 3}\right)}=2^{-\Omega\left(n^{1 / 3}\right)}\left(\begin{array}{c}
n \\
\lfloor n / 2\rfloor
\end{array}\right) .
$$

By this lemma, the number of sets in $\mathcal{F}$ in the top and bottom $n^{\prime}:=n / 2-n^{\frac{2}{3}}$ levels is $o(1)\left(\begin{array}{c}n \\ \lfloor n / 2\rfloor\end{array}\right)$, so, since we are bounding the cardinality of $\mathcal{F}$, we may assume that those levels do not contain any set from $\mathcal{F}$.

Notation. For $c \in[0,1]$, let $\tilde{c}=\min \left(c, \frac{1}{4}\right)$, and let $f(c)=1-\tilde{c}+\sqrt{\tilde{c}}$. (This is equal to $f(0, c)$ as defined in Definition 2.1.) For $A \in \mathcal{F}$, recall that $[A,[n]]$ denotes the Boolean lattice $\{S \subseteq[n]: A \subseteq S\}$. A chain of this lattice is of the form $A \subset A_{|A|+1} \subset A_{|A|+2} \subset \ldots \subset A_{n-1} \subset[n]$. (When saying just "chain", we continue to mean a maximal chain in the Boolean lattice $2^{[n]}$.) Let

$$
c(A)=\frac{1}{(n-|A|) !} \#\left\{\begin{array}{c}
\mathcal{C} \text { is a chain in }[A,[n]]: \\
\mathcal{C} \text { is MNM w.r.t. } \mathcal{F} \cap[A,[n]]
\end{array}\right\} .
$$

Further, we can assume without loss of generality that

$$
C:=\frac{1}{n !} \#\left\{\begin{array}{c}
\mathcal{C} \text { is a chain: } \mathcal{C} \cap \mathcal{F}=\emptyset \text { or } \\
\min (\mathcal{C} \cap \mathcal{F}) \text { is not minimal in } \mathcal{F}
\end{array}\right\} \geq \frac{1}{n !} \#\left\{\begin{array}{c}
\mathcal{C} \text { is a chain: } \mathcal{C} \cap \mathcal{F}=\emptyset \text { or } \\
\mathcal{C} \text { is MNM w.r.t. } \mathcal{F}
\end{array}\right\} .
$$

(If this does not hold, we can replace $\mathcal{F}$ with $\{[n] \backslash A: A \in \mathcal{F}\}$ : this family is diamond-free, has the same cardinality, and the opposite inequality holds.) Clearly $C \geq \frac{1}{n !} \#\left\{\begin{array}{c}\mathcal{C} \text { is a chain: } \\ \mathcal{C} \text { is MNM w.r.t. } \mathcal{F}\end{array}\right\}$.

$$
l(\mathcal{F})=\frac{1}{n !} \sum_{\mathcal{C} \text { is a chain }} \#(\mathcal{C} \cap \mathcal{F})=\frac{1}{n !} \sum_{A \in \mathcal{F}} \sum_{\substack{\mathcal{C} \text { is a chain } \\ A=\min (\mathcal{C} \cap \mathcal{F})}} \#(\mathcal{C} \cap \mathcal{F}),
$$

since each chain $\mathcal{C}$ will be counted when $A=\min (\mathcal{C} \cap \mathcal{F})$ - except if $\mathcal{C} \cap \mathcal{F}=\emptyset$, but then $\#(\mathcal{C} \cap \mathcal{F})=0$. Continuing,

$$
l(\mathcal{F})=\frac{1}{n !} \sum_{\substack{A \in \mathcal{F} \\
\exists \text { a chain } \mathcal{C}: A=\min (\mathcal{C} \cap \mathcal{F})}} \#\left\{\begin{array}{c}
\mathcal{C} \text { is a chain containing } A: \\
A=\min (\mathcal{C} \cap \mathcal{F})
\end{array}\right\} \frac{\sum_{\substack{\mathcal{C} \text { is a chain } \\
A=\min (\mathcal{C} \cap \mathcal{F})}} \#(\mathcal{C} \cap \mathcal{F})}{\#\left\{\begin{array}{c}
\mathcal{C} \text { is a chain containing } A:) \\
A=\min (\mathcal{C} \cap \mathcal{F})
\end{array}\right\}} .
$$

Each chain on $[A,[n]]$ can be extended to a full $2^{[n]}$-chain in $|A|$ ! ways. Furthermore, the Boolean lattice $[A,[n]]$ can be made equivalent to the Boolean lattice $2^{[n] \backslash A}$ by subtracting $A$ from each set; for $\mathcal{A} \subseteq[A,[n]]$, we denote $\mathcal{A}-A=\{S \backslash A: S \in \mathcal{A}\}$. If $A=\min (\mathcal{C} \cap \mathcal{F}), \#(\mathcal{C} \cap \mathcal{F})=\#(\mathcal{C} \cap[A,[n]] \cap \mathcal{F})$. If 
$A$ is minimal in $\mathcal{F}$ (that is, on every chain),

$$
\begin{aligned}
& \frac{\sum_{\substack{\mathcal{C} \text { is a chain } \\
A=\min (\mathcal{C} \cap \mathcal{F})}} \#(\mathcal{C} \cap \mathcal{F})}{\#\left\{\begin{array}{c}
\mathcal{C} \text { is a chain containing } A: \\
A=\min (\mathcal{C} \cap \mathcal{F})
\end{array}\right\}}=\frac{|A| ! \sum_{\mathcal{C} \text { is a chain in }[A,[n]]} \#(\mathcal{C} \cap \mathcal{F} \cap[A,[n]])}{|A| !(n-|A|) !} \\
& =\frac{\sum_{\mathcal{C} \text { is a chain in } 2^{[n] \backslash A}} \#(\mathcal{C} \cap((\mathcal{F} \cap[A,[n]])-A))}{(n-|A|) !}=l(n-|A|,(\mathcal{F} \cap[A,[n]])-A) .
\end{aligned}
$$

$(\mathcal{F} \cap[A,[n]])-A$ is diamond-free, so $((\mathcal{F} \cap[A,[n]])-A) \backslash \emptyset$ is $\Lambda$-free; and the top $n^{\prime}$ levels are assumed to be empty. Using Lemma 1.14 as well as that $\frac{1}{n^{\prime}}=\frac{1}{\Omega(n)}=o(1)$ and the subadditivity of the square root function,

$$
\begin{gathered}
l(n-|A|,((\mathcal{F} \cap[A,[n]])-A) \backslash \emptyset) \leq 1-\min \left(c(A)+\frac{1}{n^{\prime}}, \frac{1}{4}\right)+\sqrt{\min \left(c(A)+\frac{1}{n^{\prime}}, \frac{1}{4}\right)}+\frac{3}{n^{\prime}} \\
\leq f(c(A))+\sqrt{\frac{1}{n^{\prime}}}+\frac{3}{n^{\prime}}=f(c(A))+o(1),
\end{gathered}
$$

so $l(n-|A|,(\mathcal{F} \cap[A,[n]])-A) \leq 1+f(c(A))+o(1)$. Whereas if $A$ is not minimal in $\mathcal{F}$, i.e. $\exists S \in \mathcal{F}$ such that $A \supsetneqq S$, then for any chain $\mathcal{C}$ for which $\min (\mathcal{C} \cap \mathcal{F})=A$, we have $\#(\mathcal{C} \cap \mathcal{F}) \leq 2$ (otherwise $S$ and three sets in $\mathcal{C} \cap \mathcal{F}$ would form a diamond), so $\frac{\sum \underset{\mathcal{C} \text { is a chain }}{A=\min (\mathcal{C} \cap \mathcal{F})} \#(\mathcal{C} \cap \mathcal{F})}{\#\left\{\begin{array}{c}\mathcal{C} \text { is a chain through } A: \\ A=\min (\mathcal{C} \cap \mathcal{F})\end{array}\right\}} \leq 2$.

$$
\begin{aligned}
l(\mathcal{F}) & \leq \frac{1}{n !} \sum_{\substack{A \in \mathcal{F} \\
A \text { is minimal in } \mathcal{F}}} \#\{\mathcal{C} \text { is a chain containing } A\}(1+f(c(A))+o(1)) \\
& +\frac{1}{n !} \sum_{\substack{A \in \mathcal{F} \\
A \text { is not minimal in } \mathcal{F}}} \#\left\{\begin{array}{c}
\mathcal{C} \text { is a chain containing } A:\} \\
A=\min (\mathcal{C} \cap \mathcal{F})
\end{array}\right\} \cdot 2 \\
& \leq 2+\frac{1}{n !} \sum_{\substack{A \in \mathcal{F} \\
A \text { is minimal in } \mathcal{F}}} \#\{\mathcal{C} \text { is a chain containing } A\}(f(c(A))-1+o(1)) .
\end{aligned}
$$

Since $f$ is concave, we can use Jensen's inequality with the weights $\frac{\#\{\mathcal{C} \text { is a chain containing } A\}}{(1-C) n !}$ (where $A$ is minimal in $\mathcal{F}$ ). Notice that the sum of all the weights is 1 because the sum of numerators is the total number of chains $\mathcal{C}$ where $\min (\mathcal{C} \cap \mathcal{F})$ is minimal in $\mathcal{F}$, that is, $(1-C) n$ !.

$$
l(\mathcal{F}) \leq 2+(1-C)\left(f\left(\frac{\sum_{\substack{A \in \mathcal{F} \\ A \text { is minimal in } \mathcal{F}}} \#\{\mathcal{C} \text { is a chain containing } A\} c(A)}{(1-C) n !}\right)-1+o(1)\right) .
$$

$c(A)$ is the fraction of the chains containing $A$ which are MNM, so \# $\{\mathcal{C}$ is a chain containing $A\} c(A)$ is the number of MNM chains through $A$. In the numerator, each MNM chain in the whole Boolean lattice is counted once, except if the minimal element on it is not a global minimal, then it is not counted. So the numerator is less than or equal to the total number of MNM chains in the Boolean lattice, which is at most $C n$ !. Substituting, we get

$$
l(\mathcal{F}) \leq 2+(1-C)\left(f\left(\frac{n ! C}{n !(1-C)}\right)-1+o(1)\right)=1+C+(1-C) f\left(\frac{C}{1-C}\right)+o(1) .
$$

$C$ varies between 0 and 1. $\frac{C}{1-C}$ is increasing in $C$. Above $\frac{C}{1-C}=\frac{1}{4}$ (corresponding to $\left.C=\frac{1}{5}\right), f\left(\frac{C}{1-C}\right)$ is constant $\frac{5}{4}$, so $1+C+(1-C) f\left(\frac{C}{1-C}\right)=\frac{9}{4}-\frac{C}{4}$ is decreasing in C. So it is enough to take the maximum 
in the interval $\left[0, \frac{1}{5}\right]$ :

$$
\begin{aligned}
\frac{|\mathcal{F}|}{\left(\begin{array}{c}
n \\
\lfloor n / 2\rfloor
\end{array}\right)} & \leq l(\mathcal{F}) \leq \max _{C \in\left[0, \frac{1}{5}\right]}\left(1+C+(1-C)\left(1-\frac{C}{1-C}+\sqrt{\frac{C}{1-C}}\right)\right)+o(1) \\
& =\frac{\sqrt{2}+3}{2}+o(1)<2.20711+o(1) .
\end{aligned}
$$

\section{Acknowledgments}

We thank the anonymous referees for their detailed comments which helped improve the presentation of our paper. We would also like to thank Dömötör Pálvölgyi for helpful discussions concerning the 3-level version of this problem. The research of the second and third authors was supported by the National Research, Development and Innovation Office - NKFIH, grant K 116769.

\section{References}

[1] M. Axenovich, J. Manske, and R. Martin. $Q_{2}$-free families in the Boolean lattice. Order, 29:177-191, 2012.

[2] J. Balogh, P. Hu, B. Lidický, and H. Liu. Upper bounds on the size of 4- and 6-cycle-free subgraphs of the hypercube. European Journal of Combinatorics, 35:75-85, 2014.

[3] B. Bollobás. On generalized graphs. Acta Mathematica Hungarica, 16(3-4):447-452, 1965.

[4] B. Bukh. Set families with a forbidden subposet. The Electronic Journal of Combinatorics, 16(1):R142, 2009.

[5] P. Burcsi and D. Nagy. The method of double chains for largest families with excluded subposets. Electronic Journal of Graph Theory and Applications (EJGTA), 1(1), 2013.

[6] H. Chen and W.-T. Li. A note on the largest size of families of sets with a forbidden poset. Order, 31(1):137-142, 2014.

[7] É. Czabarka, A. Dutle, T. Johnston, and L. A. Székely. Abelian groups yield many large families for the diamond problem. European Journal of Mathematics, 1:320-328, 2015.

[8] A. De Bonis and G.O.H. Katona. Largest families without an $r$-fork. Order, pages 181-191, 2007.

[9] A. De Bonis, G.O.H. Katona, and K. J. Swanepoel. Largest family without $A \cup B \subset C \cap D$. Journal of Combinatorial Theory, Series A, 111(2):331-336, 2005.

[10] P. Erdôs. On a lemma of Littlewood and Offord. Bulletin of the American Mathematical Society, 51(12):898-902, 1945.

[11] G.O.H. Katona and T. Tarján. Extremal problems with excluded subgraphs in the n-cube. In Graph Theory, pages 84-93. Springer, 1983.

[12] J. R. Griggs and G.O.H. Katona. No four subsets forming an N. Journal of Combinatorial Theory, Series A, 115(4):677-685, 2008.

[13] J. R. Griggs and W.-T. Li. Progress on poset-free families of subsets. IMI USC, 2015.

[14] J. R. Griggs, W.-T. Li, and L. Lu. Diamond-free families. Journal of Combinatorial Theory, Series A, 119(2):310-322, 2012.

[15] J. R. Griggs and L. Lu. On families of subsets with a forbidden subposet. Combinatorics, Probability and Computing, 18:731-748, 2009.

[16] D. Grósz, A. Methuku, and C. Tompkins. An improvement of the general bound on the largest family of subsets avoiding a subposet. Order (2017) 34: 113. doi:10.1007/s11083-016-9390-3. 
[17] L. Kramer, R. Martin, and M. Young. On diamond-free subposets of the Boolean lattice. Journal of Combinatorial Theory, Series A, 120:545-560, 2013.

[18] L. Lu. On crown-free families of subsets. Journal of Combinatorial Theory, Series A, 126:216-231, 2014.

[19] D. Lubell. A short proof of Sperner's lemma. Journal of Combinatorial Theory, 1:299, 1966.

[20] J. Manske and J. Shen. Three layer $Q_{2}$-free families in the Boolean lattice. Order, 30:585-592, 2013.

[21] L. D. Meshalkin. Generalization of Sperner's theorem on the number of subsets of a finite set. Theory of Probability 8 Its Applications, 8(2):203-204, 1963.

[22] A. Methuku and C. Tompkins. Exact forbidden subposet results using chain decompositions of the cycle. The Electronic Journal of Combinatorics, 22(4), 2015.

[23] B. Patkós. Induced and non-induced forbidden subposet problems. The Electronic Journal of Combinatorics, 22(1), 2015.

[24] E. Sperner. Ein satz über untermengen einer endlichen menge. Mathematische Zeitschrift, 27(1):544548, 1928.

[25] H.T. Thanh. An extremal problem with excluded subposet in the boolean lattice. Order, 15(1):51-57, 1998.

[26] K. Yamamoto. Logarithmic order of free distributive lattice. Journal of the Mathematical Society of Japan, 6(3-4):343-353, 1954.

\section{Appendix A Proof of Lemma 2.2}

Points 1 and [5] are easy to see.

Proof of Point 2. It is also easy to check that $f$ is continuous at the points $x=\frac{1}{2}, c=4\left(x-x^{2}\right)^{2}$, $c=\frac{1}{4}$, and that the function is monotonously decreasing in $x$ and increasing in $c$ in each range.

$f(x, 0)=1-x ; x^{2}-2 x+1-c+\sqrt{c}$ is a concave and monotonously increasing expression in $c$. When $0<x \leq \frac{1}{2}, c \mapsto 1-x+\left(\frac{1}{4\left(x-x^{2}\right)}-1\right) c$ is the tangential line of the graph of $c \mapsto x^{2}-2 x+1-c+\sqrt{c}$ at the point $c=4\left(x-x^{2}\right)^{2}$, since both their values, and their derivatives at this point coincide. So $f$ is concave in $c$.

Since the graph of a concave function is below the tangent line at any point, we also have that for $x \in\left[0, \frac{1}{2}\right], c \in\left[0, \frac{1}{4}\right]$

$$
f(x, c) \geq x^{2}-2 x+1-c+\sqrt{c}=: \tilde{f}(x, c) .
$$

We will use this inequality in the proof of Point 3 and 4

Proof of Point 3. If $x+a=0, g(x, c, a, \tilde{a})=f(x, c)$. From now on, we assume that $x+a>0$.

We first show that $g$ is monotonously increasing in $\tilde{a}$.

$$
\left(\frac{\partial}{\partial c} f\right)(x, c)=\left(\begin{array}{ll}
\frac{1}{4\left(x-x^{2}\right)}-1 & \text { if } x \leq \frac{1}{2} \text { and } c<4\left(x-x^{2}\right)^{2} \\
-1+\frac{1}{2 \sqrt{c}} & \text { if } x \leq \frac{1}{2} \text { and } 4\left(x-x^{2}\right)^{2} \leq c \leq \frac{1}{4} \\
0 & \text { if } \frac{1}{4} \leq c \text { or } \frac{1}{2} \leq x
\end{array}\right) \leq \begin{cases}\frac{1}{4\left(x-x^{2}\right)}-1 & \text { if } x \leq \frac{1}{2} \\
0 & \text { if } \frac{1}{2} \leq x\end{cases}
$$

So,

$$
\begin{aligned}
& \frac{\partial}{\partial \tilde{a}} g(x, c, a, \tilde{a})=2(1-x-a)+(1-x-a) \cdot\left(\frac{\partial}{\partial c} f\right)\left(x+a, \frac{c-\tilde{a}(x+a)}{1-x-a}\right) \cdot \frac{\partial}{\partial \tilde{a}}\left(\frac{c-\tilde{a}(x+a)}{1-x-a}\right) \\
& \geq 2(1-x-a)-(x+a)\left(\left\{\begin{array}{ll}
\frac{1}{4\left((x+a)-(x+a)^{2}\right)}-1 & \text { if } x+a \leq \frac{1}{2} \\
0 & \text { if } \frac{1}{2} \leq x+a
\end{array}\right)\right. \\
& \geq 2(1-x-a)-(x+a)\left(\left\{\begin{array}{ll}
\frac{1}{4 \cdot \frac{1}{2}(x+a)}-1 & \text { if } x+a \leq \frac{1}{2} \\
0 & \text { if } \frac{1}{2} \leq x+a
\end{array}\right) \geq 2(1-x-a)-\left(\left\{\begin{array}{ll}
\frac{1}{2} & \text { if } x+a \leq \frac{1}{2} \\
0 & \text { if } \frac{1}{2} \leq x+a
\end{array}\right) \geq 0 .\right.\right.
\end{aligned}
$$


Therefore, from now on we assume $\tilde{a}=\min \left(a, \frac{c}{x+a}\right)$ since if $g(x, c, a, \tilde{a}) \leq f(x, c)$ holds for $\tilde{a}=$ $\min \left(a, \frac{c}{x+a}\right)$ then it also holds for any $\tilde{a} \in\left[0, \min \left(a, \frac{c}{x+a}\right)\right]$.

Case 1. First assume $a \leq \frac{c}{x+a}$ (so $\tilde{a}=a$ ), which is equivalent to $a(x+a) \leq c$ or $a \leq \frac{-x+\sqrt{x^{2}+4 c}}{2}$. Let $x^{\prime}=x+a$ and $c^{\prime}=\frac{c-a(x+a)}{1-x-a}$.

Case 1.1. When $x^{\prime} \leq \frac{1}{2}$ and $4\left(x^{\prime}-{x^{\prime}}^{2}\right)^{2} \leq c^{\prime}$, we bound $g$ from above:

$$
\begin{aligned}
g(x, c, a, a) & =a+(1-x-a) f\left(x+a, \frac{c-a(x+a)}{1-x-a}\right)+2 a(1-x-a) \\
& \leq a+(1-x-a) f\left(x+a, \frac{c}{1-x-a}\right)+2 a(1-x-a)=: \tilde{g}(x, c, a, a) .
\end{aligned}
$$

We now consider subcases based on the values of $c$ and $\frac{c}{1-x-a}$ compared to $\frac{1}{4}$. Note that $4\left(x^{\prime}-x^{\prime 2}\right)^{2} \leq$ $c^{\prime} \leq \frac{c}{1-x-a}$.

Case 1.1.1. When $c \leq \frac{c}{1-x-a} \leq \frac{1}{4}$, using (5),

$$
\begin{gathered}
g(x, c, a, a)-f(x, c) \leq \tilde{g}(x, c, a, a)-\tilde{f}(x, c)=a+(1-x-a)\left((x+a)^{2}-2(x+a)+1\right. \\
\left.-\frac{c}{1-x-a}+\frac{\sqrt{c}}{\sqrt{1-x-a}}\right)+2 a(1-x-a)-\left(x^{2}-2 x+1-c+\sqrt{c}\right) \\
=-x(1-x-2 a)+(1-x-a)(x+a)^{2}+\left(\sqrt{1-x^{\prime}}-1\right) \sqrt{c} .
\end{gathered}
$$

Thus,

$$
\frac{\partial}{\partial c}(\tilde{g}(x, c, a, a)-\tilde{f}(x, c))=\frac{\sqrt{1-x-a}-1}{2 \sqrt{c}} \leq 0 .
$$

So it is enough to check that $\tilde{g}(x, c, a, a)-\tilde{f}(x, c) \leq 0$ when $c^{\prime}=4\left(x^{\prime}-{x^{\prime}}^{2}\right)^{2}$ or, equivalently, when $c=4\left(x^{\prime}-x^{\prime 2}\right)^{2}\left(1-x^{\prime}\right)+a(x+a)$; then it is also $\leq 0$ for bigger $c$. First some auxiliary calculations:

$$
\begin{gathered}
\sqrt{1-x^{\prime}}-1 \leq \sqrt{1-x^{\prime}+\frac{x^{\prime 2}}{4}}-1=-\frac{x^{\prime}}{2} \leq 0 . \\
4\left(x^{\prime}-{x^{\prime}}^{2}\right)^{2} \leq 4{x^{\prime}}^{2} \text { and } 4\left(x^{\prime}-{x^{\prime}}^{2}\right)^{2} \leq 4 \cdot\left(\frac{1}{4}\right)^{2}=\frac{1}{4}, \quad \text { so } \\
4\left(x^{\prime}-{x^{\prime}}^{2}\right)^{2} \leq \min \left(4 x^{\prime 2}, \frac{1}{4}\right) \leq x^{\prime} .
\end{gathered}
$$

So,

$$
\begin{gathered}
4\left(x^{\prime}-{x^{\prime}}^{2}\right)^{2}\left(1-x^{\prime}\right)+a(x+a) \geq 4\left(x^{\prime}-{x^{\prime}}^{2}\right)^{2}\left(1-x^{\prime}\right)+4\left(x^{\prime}-{x^{\prime}}^{2}\right)^{2} a \\
=\left(2\left(x^{\prime}-{x^{\prime}}^{2}\right)\right)^{2}(1-x) \geq\left(2\left(x^{\prime}-{x^{\prime}}^{2}\right)\right)^{2}(1-x)^{2} .
\end{gathered}
$$

Putting $c=4\left(x^{\prime}-x^{\prime 2}\right)^{2}\left(1-x^{\prime}\right)+a(x+a)$ in (7) and then using (8) and (10),

$$
\begin{aligned}
\tilde{g}(x, c, a, a)-\tilde{f}(x, c) & =-x(1-x-2 a)+(1-x-a)(x+a)^{2} \\
& +\left(\sqrt{1-x^{\prime}}-1\right) \sqrt{4\left(x^{\prime}-x^{\prime 2}\right)^{2}\left(1-x^{\prime}\right)+a(x+a)} \\
& \leq-x(1-x-2 a)+(1-x-a)(x+a)^{2}-x^{\prime}\left(x^{\prime}-x^{\prime 2}\right)(1-x) \\
& =-x\left[(1-x-2 a)-(1-x-a)(x+a)^{2}\right] \leq-x\left[(1-x-2 a)-(1-x-a) \frac{1}{4}\right] \\
& =-x\left(\frac{3-3 x-7 a}{4}\right),
\end{aligned}
$$


which is $\leq 0$ when $a \leq \frac{3-3 x-3 a}{4}=\frac{3-3 x^{\prime}}{4}$. Assume $a>\frac{3-3 x^{\prime}}{4}$. Since $x^{\prime} \geq a, x^{\prime}>\frac{3}{7}$ (and we have also assumed $\left.\frac{1}{2} \geq x^{\prime}\right)$; and

$$
\begin{aligned}
c & =4\left(x^{\prime}-{x^{\prime}}^{2}\right)^{2}\left(1-x^{\prime}\right)+a(x+a)>4\left(x^{\prime}-x^{\prime 2}\right)^{2}\left(1-x^{\prime}\right)+\frac{\left(3-3 x^{\prime}\right) x^{\prime}}{4} \\
& >4 \cdot\left(\frac{3}{7}-\left(\frac{3}{7}\right)^{2}\right)^{2}\left(1-\frac{1}{2}\right)+\frac{\left(3-3 \cdot \frac{1}{2}\right) \cdot \frac{3}{7}}{4}=\frac{5391}{19208}>\frac{1}{4}
\end{aligned}
$$

contrary to our assumption that $c \leq \frac{c}{1-x-a} \leq \frac{1}{4}$.

Case 1.1.2. When $c \leq \frac{1}{4}<\frac{c}{1-x-a}$ (and recall $4\left(x^{\prime}-x^{\prime 2}\right)^{2}\left(1-x^{\prime}\right)+a(x+a) \leq c$ ), using (5),

$$
\begin{aligned}
g(x, c, a, a)-f(x, c) & \leq \tilde{g}(x, c, a, a)-\tilde{f}(x, c)=a+(1-x-a)\left((x+a)^{2}-2(x+a)+1.25\right) \\
& +2 a(1-x-a)-\left(x^{2}-2 x+1-c+\sqrt{c}\right) \\
& =-x(1-x-2 a)+(1-x-a)\left((x+a)^{2}+0.25\right)+c-\sqrt{c} .
\end{aligned}
$$

Case 1.1.2.1. If $4\left(x^{\prime}-{x^{\prime}}^{2}\right)^{2}\left(1-x^{\prime}\right)+a(x+a) \leq \frac{1}{4}(1-x-a)$ (which is $\left.<c\right)$, since $t-\sqrt{t}$ is decreasing in $0 \leq t \leq \frac{1}{4}$, replacing $c$ by $\frac{1}{4}(1-x-a)$ in (11), we get

$$
\begin{gathered}
g(x, c, a, a)-f(x, c) \leq-x(1-x-2 a)+(1-x-a)\left((x+a)^{2}+0.25\right)+\frac{1}{4}(1-x-a) \\
-\sqrt{\frac{1}{4}(1-x-a)}=\tilde{g}\left(x, \frac{1}{4}(1-x-a), a, a\right)-\tilde{f}\left(x, \frac{1}{4}(1-x-a)\right) \leq 0
\end{gathered}
$$

as it falls in Case 1.1.1. above.

Case 1.1.2.2. If $\frac{1}{4}(1-x-a) \leq 4\left(x^{\prime}-x^{\prime 2}\right)^{2}\left(1-x^{\prime}\right)+a(x+a)$ (which is $\left.\leq c\right)$, again, by (11) we have,

$$
\begin{aligned}
& g(x, c, a, a)-f(x, c) \leq-x(1-x-2 a)+(1-x-a)\left((x+a)^{2}+0.25\right) \\
& +4\left(x^{\prime}-{x^{\prime}}^{2}\right)^{2}\left(1-x^{\prime}\right)+a(x+a)-\sqrt{4\left(x^{\prime}-x^{\prime 2}\right)^{2}\left(1-x^{\prime}\right)+a(x+a) .}
\end{aligned}
$$

Let $b=\max \left(4\left(x^{\prime}-{x^{\prime}}^{2}\right)^{2}\left(1-x^{\prime}\right)+{x^{\prime}}^{2}-\frac{1}{4}, 0\right)$. Now some auxiliary calculations follow. Since $\frac{\mathrm{d}}{\mathrm{d} t} \sqrt{t}=\frac{1}{2 \sqrt{t}}$, and by (10),

$$
\begin{gathered}
\sqrt{4\left(x^{\prime}-{x^{\prime}}^{2}\right)^{2}\left(1-x^{\prime}\right)+{x^{\prime}}^{2}-b}-\sqrt{4\left(x^{\prime}-{x^{\prime}}^{2}\right)^{2}\left(1-x^{\prime}\right)+a(x+a)} \\
\leq \frac{1}{2 \sqrt{4\left(x^{\prime}-{x^{\prime}}^{2}\right)^{2}\left(1-x^{\prime}\right)+a(x+a)}}(x(x+a)-b) \leq \frac{1}{4\left(x^{\prime}-x^{\prime 2}\right)(1-x)}(x(x+a)-b) . \\
(1-a) \cdot 4\left(x^{\prime}-{x^{\prime}}^{2}\right)(1-x) \geq 4\left(x^{\prime}-{x^{\prime}}^{2}\right)\left(1-x^{\prime}\right)=4\left(1-x^{\prime}\right)^{2} x^{\prime} \geq 4 \cdot\left(\frac{1}{2}\right)^{2} x^{\prime}=x+a .
\end{gathered}
$$

So,

$$
\begin{gathered}
-x(1-x-2 a)+\left(\frac{1}{4\left(x^{\prime}-x^{\prime 2}\right)(1-x)}-1\right)(x(x+a)-b) \\
\leq-x(1-x-2 a)+\left(\frac{1-a}{x+a}-1\right) x(x+a)=0 .
\end{gathered}
$$

By (9), $4\left(x^{\prime}-x^{\prime 2}\right)^{2} \leq x^{\prime}$, so

$$
\begin{gathered}
4\left(x^{\prime}-{x^{\prime}}^{2}\right)^{2}\left(1-x^{\prime}\right)+{x^{\prime}}^{2} \geq 4\left(x^{\prime}-{x^{\prime}}^{2}\right)^{2}\left(1-x^{\prime}\right)+4\left(x^{\prime}-{x^{\prime}}^{2}\right)^{2} x^{\prime}=\left(2\left(x^{\prime}-{x^{\prime}}^{2}\right)\right)^{2} . \\
4\left(x^{\prime}-{x^{\prime}}^{2}\right)^{2}\left(1-x^{\prime}\right)+x^{\prime 2}-b \geq\left(2\left(x^{\prime}-x^{\prime 2}\right)\right)^{2}
\end{gathered}
$$


since if $b>0,4\left(x^{\prime}-{x^{\prime}}^{2}\right)^{2}\left(1-x^{\prime}\right)+{x^{\prime}}^{2}-b=\frac{1}{4} \geq\left(2\left(\frac{1}{4}-\left(\frac{1}{2}-x^{\prime}\right)^{2}\right)\right)^{2}=\left(2\left(x^{\prime}-x^{\prime 2}\right)\right)^{2}$ (if $\mathrm{b}=$ 0 , then it holds by (15)). Now using (13) in (12), and then using (14) and (16) and that $t-\sqrt{t}$ is decreasing in $0 \leq t \leq 1 / 4$, we get

$$
\begin{gathered}
g(x, c, a, a)-f(x, c) \leq(1-x-a)\left((x+a)^{2}+0.25\right)+4\left(x^{\prime}-x^{\prime 2}\right)^{2}\left(1-x^{\prime}\right)+x^{\prime 2}-b \\
-\sqrt{4\left(x^{\prime}-x^{\prime 2}\right)^{2}\left(1-x^{\prime}\right)+x^{\prime 2}-b}-x(1-x-2 a)+\left(\frac{1}{4\left(x^{\prime}-x^{\prime 2}\right)(1-x)}-1\right)(x(x+a)-b) \\
\leq(1-x-a)\left((x+a)^{2}+0.25\right)+\left(2\left(x^{\prime}-x^{\prime 2}\right)\right)^{2}-2\left(x^{\prime}-x^{\prime 2}\right) \\
=4\left(x^{\prime}-\frac{1}{4}\right)\left(x^{\prime}-\frac{1}{2}\right)^{2}\left(x^{\prime}-1\right),
\end{gathered}
$$

which is $\leq 0$ when $\frac{1}{4} \leq x^{\prime} \leq 1$. When $x^{\prime}<\frac{1}{4}$, we show that this subcase cannot hold:

$$
\begin{gathered}
4\left(x^{\prime}-{x^{\prime}}^{2}\right)^{2}\left(1-x^{\prime}\right)+a(x+a)-\frac{1}{4}(1-x-a)<\left(4 \cdot\left(\frac{1}{4}-\left(\frac{1}{4}\right)^{2}\right)^{2}-\frac{1}{4}\right)\left(1-x^{\prime}\right)+{x^{\prime 2}}^{2} \\
=-\frac{7}{64}\left(1-x^{\prime}\right)+{x^{\prime}}^{2}<-\frac{1}{12}\left(1-x^{\prime}\right)+{x^{\prime}}^{2}=\left(x^{\prime}-\frac{1}{4}\right)\left(x^{\prime}+\frac{1}{3}\right)<0 .
\end{gathered}
$$

Case 1.1.3. When $\frac{1}{4} \leq c$ (which is $\left.<\frac{c}{1-x-a}\right)$,

$$
\begin{aligned}
g(x, c, a, a)-f(x, c) & \leq \tilde{g}(x, c, a, a)-f(x, c)=a+(1-x-a)\left((x+a)^{2}-2(x+a)+1.25\right) \\
& +2 a(1-x-a)-\left(x^{2}-2 x+1.25\right)=\tilde{g}\left(x, \frac{1}{4}, a, a\right)-\tilde{f}\left(x, \frac{1}{4}\right) \leq 0,
\end{aligned}
$$

as it falls in Case 1.1.1. or 1.1.2. above.

Case 1.2. When $x^{\prime} \leq \frac{1}{2}$ and $c^{\prime} \leq 4\left(x^{\prime}-{x^{\prime}}^{2}\right)^{2}$,

$$
g(x, c, a, a)=a+(1-x-a)\left(1-x^{\prime}+\left(\frac{1}{4\left(x^{\prime}-x^{\prime 2}\right)}-1\right) c^{\prime}\right)+2 a(1-x-a),
$$

which is linear in $c^{\prime}$, so also in $c . f(x, c)$ is concave in $c$, so it is enough to check that $g$ is smaller than $f$ in the ends of the interval $c \in\left[a(a+x), 4\left(x^{\prime}-x^{\prime 2}\right)^{2}\left(1-x^{\prime}\right)+a(x+a)\right]$ :

$$
\begin{aligned}
g(x, a(a+x), a, a) & =a+(1-x-a)^{2}+2 a(1-x-a) \\
& \leq a+(1-x-a)^{2}+2 a(1-x-a)+\frac{2 a x\left(\frac{1}{2}-x-a+\left(1-\frac{a}{2}\right) x\right)}{\sqrt{a(x+a)}+a x+a} \\
& =x^{2}-2 x+1-a(x+a)+\sqrt{a(x+a)} \leq f(x, a(x+a)),
\end{aligned}
$$

since $\frac{1}{2}-x-a \geq 0$ and $a(x+a) \leq{x^{\prime}}^{2} \leq \frac{1}{4}$, and using (5). Whereas the higher end of the interval was handled above in Case 1.1. since $f$ is continuous.

Case 1.3. Finally, when $\frac{1}{2} \leq x^{\prime}$,

$$
g(x, c, a, a)=a+(1-x-a)^{2}+2 a(1-x-a)=x^{2}-2 x+1-a^{2}+a .
$$

If $x \leq \frac{1}{2}$, let $\tilde{c}=\min \left(c, \frac{1}{4}\right)$. Since $c \geq a(x+a) \geq a^{2}$, and by (5)

$$
g(x, c, a, a)=x^{2}-2 x+1-a^{2}+a \leq x^{2}-2 x+1-\tilde{c}+\sqrt{\tilde{c}} \leq f(x, c) .
$$

If $\frac{1}{2} \leq x$, then $a \leq 1-x \leq \frac{1}{2}$. $-a^{2}+a$ is monotonously increasing in $a \in\left[0, \frac{1}{2}\right]$, so

$$
g(x, c, a, a)=x^{2}-2 x+1-a^{2}+a \leq x^{2}-2 x+1-(1-x)^{2}+1-x=1-x \leq f(x, c),
$$

by Point 5 
Case 2. Now consider $a \geq \frac{c}{x+a}$, that is, $a \geq \frac{-x+\sqrt{x^{2}+4 c}}{2}$. Then $\tilde{a}=\frac{c}{x+a} \leq \frac{c}{x+\frac{-x+\sqrt{x^{2}+4 c}}{2}}=\frac{-x+\sqrt{x^{2}+4 c}}{2}$, and $\frac{c-\tilde{a}(x+a)}{1-x-a}=0$, so $f\left(x+a, \frac{c-\tilde{a}(x+a)}{1-x-a}\right)=1-x-a$.

$$
g(x, c, a, \tilde{a})=a+(1-x-a)^{2}+2 \frac{c}{x+a}(1-x-a) \leq a+(1-x-a)^{2}+\left(-x+\sqrt{x^{2}+4 c}\right)(1-x-a)
$$

which is quadratic in $a$ with a positive leading coefficient, so its maximum is at one end of the interval $\left[\frac{-x+\sqrt{x^{2}+4 c}}{2}, 1-x\right] \cdot a=\frac{-x+\sqrt{x^{2}+4 c}}{2}$ (i.e., $a=\frac{c}{x+a}=\tilde{a}$ ) was handled above in Case 1. If $a=1-x$, the right side of the inequality equals $1-x$ which is $\leq f(x, c)$ by Point 5 ,

Proof of Point 4. If $x+a=0, h(x, c, a, \tilde{a})=f(x, c)$. From now on, we assume that $x+a>0$.

We first show that $h$ is monotonously increasing in $\tilde{a}$. Using (6) and a calculation similar to the one in the proof of Point 3, we have

$$
\begin{gathered}
\frac{\partial}{\partial \tilde{a}} h(x, c, a, \tilde{a})=2(1-x-a)+(1-x-a) \cdot\left(\frac{\partial}{\partial c} f\right)\left(x+a, \frac{c-(x+\tilde{a})(x+a)}{1-x-a}\right) \\
\cdot \frac{\partial}{\partial \tilde{a}}\left(\frac{c-(x+\tilde{a})(x+a)}{1-x-a}\right) \geq 2(1-x-a)-(x+a)\left(\left\{\begin{array}{ll}
\frac{1}{4\left((x+a)-(x+a)^{2}\right)}-1 & \text { if } x+a \leq \frac{1}{2} \\
0 & \text { if } \frac{1}{2} \leq x+a
\end{array}\right) \geq 0 .\right.
\end{gathered}
$$

Therefore, from now on we assume $\tilde{a}=\min \left(a, \frac{c}{x+a}-x\right)$.

Case 1. First assume $a \leq \frac{c}{x+a}-x$ (so $\tilde{a}=a$ ), which is equivalent to $(x+a)^{2} \leq c$ or $a \leq \sqrt{c}-x$. Let $x^{\prime}=x+a$ and $c^{\prime}=\frac{c-(x+a)^{2}}{1-x-a}$.

Case 1.1. When $x^{\prime} \leq \frac{1}{2}$ and $4\left(x^{\prime}-{x^{\prime}}^{2}\right)^{2} \leq c^{\prime}$, we bound $h$ from above:

$$
\begin{aligned}
& h(x, c, a, a)=a+(1-x-a) f\left(x+a, \frac{c-(x+a)^{2}}{1-x-a}\right)+2 a(1-x-a)+x-3 x(x+a) \\
& \leq a+(1-x-a) f\left(x+a, \frac{c}{1-x-a}\right)+2 a(1-x-a)+x-3 x(x+a)=: \tilde{h}(x, c, a, a) .
\end{aligned}
$$

We now consider subcases based on the values of $c$ and $\frac{c}{1-x-a}$ compared to $\frac{1}{4}$. Note that $4\left(x^{\prime}-{x^{\prime}}^{2}\right)^{2} \leq$ $c^{\prime} \leq \frac{c}{1-x-a}$.

Case 1.1.1. When $c \leq \frac{c}{1-x-a} \leq \frac{1}{4}$, using (5),

$$
\begin{gathered}
h(x, c, a, a)-f(x, c) \leq \tilde{h}(x, c, a, a)-\tilde{f}(x, c)=a+(1-x-a)\left((x+a)^{2}-2(x+a)+1\right. \\
\left.-\frac{c}{1-x-a}+\frac{\sqrt{c}}{\sqrt{1-x-a}}\right)+2 a(1-x-a)+x-3 x(x+a)-\left(x^{2}-2 x+1-c+\sqrt{c}\right) \\
=-2 x^{2}-x a+(1-x-a)(x+a)^{2}+\left(\sqrt{1-x^{\prime}}-1\right) \sqrt{c} . \\
\frac{\partial}{\partial c}(\tilde{h}(x, c, a, a)-\tilde{f}(x, c))=\frac{\sqrt{1-x-a}-1}{2 \sqrt{c}} \leq 0 .
\end{gathered}
$$

So it is enough to check that $\tilde{h}(x, c, a, a)-\tilde{f}(x, c) \leq 0$ when $c^{\prime}=4\left(x^{\prime}-x^{\prime 2}\right)^{2}$ or, equivalently, when $c=4\left(x^{\prime}-x^{\prime 2}\right)^{2}\left(1-x^{\prime}\right)+x^{\prime 2}$; then it is also $\leq 0$ for bigger $c$. As seen in (15) and (8) in the proof of Point 3, $4\left(x^{\prime}-{x^{\prime}}^{2}\right)^{2}\left(1-x^{\prime}\right)+{x^{\prime}}^{2} \geq\left(2\left(x^{\prime}-{x^{\prime}}^{2}\right)\right)^{2}$, and $\sqrt{1-x^{\prime}}-1 \leq-\frac{x^{\prime}}{2} \leq 0$. Putting $c=4\left(x^{\prime}-{x^{\prime}}^{2}\right)^{2}\left(1-x^{\prime}\right)+{x^{\prime}}^{2}$ in (18) and using these inequalities, we get

$$
\begin{aligned}
\tilde{h}(x, c, a, a)-\tilde{f}(x, c) & =-2 x^{2}-x a+(1-x-a)(x+a)^{2} \\
& +\left(\sqrt{1-x^{\prime}}-1\right) \sqrt{4\left(x^{\prime}-x^{\prime 2}\right)^{2}\left(1-x^{\prime}\right)+x^{\prime 2}} \\
& \leq-2 x^{2}-x a+(1-x-a)(x+a)^{2}-x^{\prime}\left(x^{\prime}-x^{\prime 2}\right)=-2 x^{2}-x a \leq 0 .
\end{aligned}
$$


Case 1.1.2. When $c \leq \frac{1}{4}<\frac{c}{1-x-a}\left(\right.$ and recall $\left.\left(2\left(x^{\prime}-{x^{\prime}}^{2}\right)\right)^{2} \leq 4\left(x^{\prime}-{x^{\prime}}^{2}\right)^{2}\left(1-x^{\prime}\right)+{x^{\prime}}^{2} \leq c\right)$, using (5),

$$
\begin{aligned}
h(x, c, a, a)-f(x, c) & \leq \tilde{h}(x, c, a, a)-\tilde{f}(x, c)=a+(1-x-a)\left((x+a)^{2}-2(x+a)+1.25\right) \\
& +2 a(1-x-a)+x-3 x(x+a)-\left(x^{2}-2 x+1-c+\sqrt{c}\right) \\
& =-2 x^{2}-x a+(1-x-a)\left((x+a)^{2}+0.25\right)+c-\sqrt{c} .
\end{aligned}
$$

Case 1.1.2.1. If $4\left(x^{\prime}-{x^{\prime 2}}^{2}\right)^{2}\left(1-x^{\prime}\right)+{x^{\prime}}^{2} \leq \frac{1}{4}(1-x-a)$ (which is $\left.<c\right)$, since $t-\sqrt{t}$ is decreasing in $0 \leq t \leq \frac{1}{4}$, replacing $c$ by $\frac{1}{4}(1-x-a)$ in (19), we get

$$
\begin{aligned}
h(x, c, a, a)-f(x, c) & \leq-2 x^{2}-x a+(1-x-a)\left((x+a)^{2}+0.25\right)+\frac{1}{4}(1-x-a) \\
& -\sqrt{\frac{1}{4}(1-x-a)}=\tilde{h}\left(x, \frac{1}{4}(1-x-a), a, a\right)-\tilde{f}\left(x, \frac{1}{4}(1-x-a)\right) \leq 0,
\end{aligned}
$$

as it falls in Case 1.1.1. above.

Case 1.1.2.2. If $\frac{1}{4}(1-x-a) \leq 4\left(x^{\prime}-{x^{\prime}}^{2}\right)^{2}\left(1-x^{\prime}\right)+{x^{\prime}}^{2}($ which is $\leq c)$, again, by (19) (and since $\left.-2 x^{2}-x a \leq 0\right)$, we get

$$
\begin{aligned}
h(x, c, a, a)-f(x, c) & \leq(1-x-a)\left((x+a)^{2}+0.25\right)+\left(2\left(x^{\prime}-{x^{\prime}}^{2}\right)\right)^{2}-2\left(x^{\prime}-{x^{\prime}}^{2}\right) \\
& =4\left(x^{\prime}-\frac{1}{4}\right)\left(x^{\prime}-\frac{1}{2}\right)^{2}\left(x^{\prime}-1\right),
\end{aligned}
$$

which is $\leq 0$ when $\frac{1}{4} \leq x^{\prime} \leq 1$. When $x^{\prime}<\frac{1}{4}$, we show that this subcase cannot hold:

$$
4\left(x^{\prime}-x^{\prime 2}\right)^{2}\left(1-x^{\prime}\right)+x^{\prime 2}-\frac{1}{4}(1-x-a)<\left(4 \cdot\left(\frac{1}{4}-\left(\frac{1}{4}\right)^{2}\right)^{2}-\frac{1}{4}\right)\left(1-x^{\prime}\right)+x^{\prime 2}<0,
$$

like in (17) in the proof of Point 3 .

Case 1.1.3. When $\frac{1}{4} \leq c$ (which is $\left.<\frac{c}{1-x-a}\right)$,

$$
\begin{gathered}
h(x, c, a, a)-f(x, c) \leq \tilde{h}(x, c, a, a)-f(x, c)=a+(1-x-a)\left((x+a)^{2}-2(x+a)+1.25\right) \\
\quad+2 a(1-x-a)+x-3 x(x+a)-\left(x^{2}-2 x+1.25\right)=\tilde{h}\left(x, \frac{1}{4}, a, a\right)-\tilde{f}\left(x, \frac{1}{4}\right) \leq 0,
\end{gathered}
$$

as it falls in Case 1.1.1. or 1.1.2. above.

Case 1.2. When $x^{\prime} \leq \frac{1}{2}$ and $c^{\prime} \leq 4\left(x^{\prime}-x^{\prime 2}\right)^{2}$,

$$
h(x, c, a, a)=a+(1-x-a)\left(1-x^{\prime}+\left(\frac{1}{4\left(x^{\prime}-x^{\prime 2}\right)}-1\right) c^{\prime}\right)+2 a(1-x-a)+x-3 x(x+a),
$$

which is linear in $c^{\prime}$, so also in $c$. $f(x, c)$ is concave in $c$, so it is enough to check that $h$ is smaller than $f$ in the ends of the interval $c \in\left[(x+a)^{2}, 4\left(x^{\prime}-{x^{\prime}}^{2}\right)^{2}\left(1-x^{\prime}\right)+{x^{\prime}}^{2}\right]$ :

$$
\begin{aligned}
h\left(x,(x+a)^{2}, a, a\right) & =a+(1-x-a)^{2}+2 a(1-x-a)+x-3 x(x+a) \\
& \leq a+(1-x-a)^{2}+2 a(1-x-a)+x-3 x(x+a)+x(2 x+a) \\
& =x^{2}-2 x+1-(x+a)^{2}+(x+a) \leq f\left(x,(x+a)^{2}\right)
\end{aligned}
$$

since $(x+a)^{2}=x^{\prime 2} \leq \frac{1}{4}$, and using (5). Whereas the higher end of the interval was handled above in Case 1.1. since $f$ is continuous.

Case 1.3. Finally, when $\frac{1}{2} \leq x^{\prime}, c \geq(x+a)^{2} \geq \frac{1}{4}$, so

$$
h(x, c, a, a)=a+(1-x-a)^{2}+2 a(1-x-a)+x-3 x(x+a) \leq x^{2}-2 x+1-(x+a)^{2}+(x+a) .
$$


If $x \leq \frac{1}{2}$, then

$$
h(x, c, a, a) \leq x^{2}-2 x+1-(x+a)^{2}+(x+a) \leq x^{2}-2 x+1.25=f(x, c) .
$$

If $\frac{1}{2} \leq x$, then $-(x+a)^{2}+(x+a)$ is monotonously decreasing in $a$, so

$$
h(x, c, a, a) \leq x^{2}-2 x+1-(x+a)^{2}+(x+a) \leq x^{2}-2 x+1-x^{2}+x=1-x=f(x, c) .
$$

Case 2. Now we consider $a \geq \frac{c}{x+a}-x$, that is, $a \geq \sqrt{c}-x$. Then $\tilde{a}=\min \left(a, \frac{c}{x+a}-x\right)=\frac{c}{x+a}-x \leq$ $\sqrt{c}-x$, and $\frac{c-(x+\tilde{a})(x+a)}{1-x-a}=0$, so $f\left(x+a, \frac{c-(x+\tilde{a})(x+a)}{1-x-a}\right)=1-x-a$.

$$
\begin{aligned}
h(x, c, a, \tilde{a}) & =a+(1-x-a)^{2}+2\left(\frac{c}{x+a}-x\right)(1-x-a)+x-3 x(x+a) \\
& \leq a+(1-x-a)^{2}+2(\sqrt{c}-x)(1-x-a)+x-3 x(x+a),
\end{aligned}
$$

which is quadratic in $a$ with a positive leading coefficient, so its maximum is at one end of the interval $[\sqrt{c}-x, 1-x] . a=\sqrt{c}-x$ (i.e., $a=\frac{c}{x+a}-x=\tilde{a}$ ) was handled above in Case 1. If $a=1-x$, the right side of the inequality equals $1-3 x$ which is $\leq f(x, c)$ by Point $[5$ 